\title{
Correspondence
}

\section{Cross-Layer Network Lifetime Maximization in Interference-Limited WSNs}

\author{
Halil Yetgin, Student Member, IEEE, \\ Kent Tsz Kan Cheung, Student Member, IEEE, \\ Mohammed El-Hajjar, Member, IEEE, and \\ Lajos Hanzo, Fellow, IEEE
}

7 Abstract-In wireless sensor networks (WSNs), the network lifetime 8 (NL) is a crucial metric since the sensor nodes usually rely on lim9 ited energy supply. In this paper, we consider the joint optimal design 10 of the physical, medium access control (MAC), and network layers to 11 maximize the NL of the energy-constrained WSN. The problem of NL 12 maximization can be formulated as a nonlinear optimization problem 13 encompassing the routing flow, link scheduling, transmission rate, and 14 power allocation operations for all active time slots (TSs). The resultant 15 nonconvex rate constraint is relaxed by employing an approximation of 16 the signal-to-interference-plus-noise ratio (SINR), which transforms the 17 problem to a convex one. Hence, the resultant dual problem may be 18 solved to obtain the optimal solution to the relaxed problem with a zero 19 duality gap. Therefore, the problem is formulated in its Lagrangian form, 20 and the Karush-Kuhn-Tucker (KKT) optimality conditions are employed 21 for deriving analytical expressions of the globally optimal transmission 22 rate and power allocation variables for the network topology considered.

23 The nonlinear Gauss-Seidel algorithm is adopted for iteratively updating

24 the rate and power allocation variables using these expressions until 25 convergence is attained. Furthermore, the gradient method is applied for 26 updating the dual variables in each iteration. Using this approach, the 27 maximum NL, the energy dissipation per node, the average transmission 28 power per link, and the lifetime of all nodes in the network are evaluated 29 for a given source rate and fixed link schedule under different channel 30 conditions.

31 Index Terms-Author, please supply index terms/keywords for your 32 paper. To download the IEEE Taxonomy go to http://www.ieee.org/ 33 documents/taxonomy_v101.pdf.

\section{NOMENCLATURE}

- Number of nodes: $V=10$.

- Total number of TSs per link: $N=18$.

- Path-loss exponent: $m=4$.

- Euclidean distance between consecutive nodes: $d[\mathrm{~m}]=1$.

- Maximum affordable transmit power per node: $\left(P_{v}\right)_{\max } \cdot[\mathrm{W}]=50$.

- Spatially periodic link scheduling parameter: $T=\{3,4,5,6$, $7,8,9\}$.

Manuscript received November 29, 2013; revised August 1, 2014; accepted September 20, 2014. This work was supported in part by the Republic of Turkey Ministry of National Education, by the Industrial Company Members of the Mobile VCE, by the U.K. Engineering and Physical Sciences Research Council, and by the Research Councils U.K. through the India-U.K. Advanced Technology Center of the European Union under the auspices of the Concerto Project and of the European Research Council's Senior Research Fellow Grant. The review of this paper was coordinated by Prof. S. Chen.

The authors are with the School of Electronics and Computer Science, University of Southampton, Southampton SO17 1BJ, U.K. (e-mail: hy3g09@ ecs.soton.ac.uk; ktkc106@ecs.soton.ac.uk; meh@ecs.soton.ac.uk; lh@ecs. soton.ac.uk).

Color versions of one or more of the figures in this paper are available online at http://ieeexplore.ieee.org.

Digital Object Identifier 10.1109/TVT.2014.2360361
- Initial battery energy per node: $E_{v}[\mathrm{~J}]=5000$.

43

- Spectral noise power density: $N 0[\mathrm{dBm} / \mathrm{Hz}]=1$.

- Power amplifier inefficiency: $\alpha=0.01$ [26].

- Set of all directed links: $\mathcal{L}$.

- A directed link spanning from transmitter $i$ to receiver $j: l_{i, j}$

- Set of all sensor nodes: $\mathcal{V}$.

- Network topology incidence matrix: A.

- Emerging link of node $v: l \in \mathcal{O}(v)$.

- Incoming link of node $v: l \in \mathcal{I}(v)$.

- Network-channel-gain matrix: G.

- Fading gain of the link between transmitter $i$ and receiver $j: 53$ $H_{i, j}=\left|h_{i, j}\right|^{2}$.

- NL: $T_{\text {net }}$.

- Reciprocal of NL: $z$.

- Transmission rate of link $l$ in TS $n: r_{l, n}$.

- Transmit power of link $l$ in TS $n: P_{l, n}$.

- Logarithm of the transmit power of link $l$ in TS $n: Q_{l, n}=59$ $\log \left(P_{l, n}\right)$.

- A set of dual variables for energy conservation constraint in 61 (5): $\Omega$.

- A set of dual variables for transmission rate constraint in (4): $\Psi .63$

- A set of dual variables for transmit power constraint in (6): $\vartheta$. 64

- A set of dual variables for flow constraint in (3): $\boldsymbol{\mu}$.

- Convergence tolerance of the iterative algorithm: $\epsilon=10^{-5}$. 66

\section{INTRODUCTION}

A wireless sensor network (WSN) is composed of a large number 68 of nodes that monitor physical and environmental conditions and pass 69 their accumulated data through the network to a sink node. There are 70 numerous attractive applications for WSNs, including, for example, 71 designing intelligent highways, controlling air pollution, providing 72 remote health assistance for disabled or elderly people, monitoring 73 river level variations, etc. Each of these applications may be composed 74 of many sensor nodes, each of which consumes considerable amount 75 of energy with sensing, communication, and data processing activities. 76 Since each sensor node drains its limited energy supply as time elapses, 77 the network lifetime (NL) is a crucial metric for these applications and 78 has a major impact on the achievable performance of WSNs. Hence, 79 we aim for analyzing and optimizing the NL of the WSNs under 80 different channel conditions.

The NL defines the total amount of time during which the network is 82 capable of maintaining its full functionality and/or achieves particular 83 objectives during its operation, as exemplified in [1] and [2]. Specifi- 84 cally, the authors of [3]-[5] defined the expiration of the NL as the time 85 instant at which a certain number of nodes in the network depleted 86 their batteries. As a further example, the NL was defined in [6] as 87 the lifetime of the specific sensor node associated with the highest 88 energy consumption rate, whereas the authors of [7]-[9] considered 89 the lifetime of the network to be expired at the particular instant, when 90 the first node's battery was depleted. The NL in [8] was also defined 91 as the instant when the first data collection failure occurred. In this 92 paper, the NL is deemed to be expired, when at least one of the nodes 93 fails due to its discharged battery. Therefore, extending the lifetime 94 of a single node becomes an important and challenging task due to 95 the battery-dependent characteristics of the wireless sensor nodes. 96 This common NL definition is used in this paper since we consider 97 
98 a network of linearly connected sensor nodes, where a single node's 99 failure may destroy the entire string topology of nodes and, hence, 100 the information of the source cannot be relayed to the sink. When 101 considering the energy dissipated at a sensor node, the battery life 102 is predominantly related to the node's communication activity, where 103 the transmission rate and power must be optimized, while taking into 104 account the battery capacity, the efficiency of the power amplifiers, the 105 receiver and transmitter circuit energy consumption, and other physical 106 layer parameters, including the modulation and coding schemes, the 107 attainable coding gain, the path loss, and so on.

108 It is widely recognized that transmission at a high transmission rate 109 requires the use of high transmit power, which potentially leads to 110 strong interference among the transmission links [10]. Therefore, the 111 battery depletion of an individual sensor node may become inevitable; 112 hence, the NL may be reduced. However, in large networks, spatial 113 reuse may be adopted for improving the attainable transmission rates at 114 the cost of imposing interference on the network [11]. In this case, link 115 scheduling [12] and multiple-access schemes [13] play a significant 116 role in coordinating the resultant interference. More explicitly, we will 117 demonstrate that scheduling weakly interfering links simultaneously 118 allows the network to maintain a given sum rate at a reduced per-node 119 transmit power, which hence extends the battery life of the nodes and 120 the NL [10]. This is one of the methods routinely employed for taking 121 advantage of spatial reuse to control the level of interference imposed 122 on the network [11]. This method extends the NL since mitigating 123 the interference imposed implies that each transmission requires less 124 power. Therefore, intelligent scheduling should carefully balance the 125 number of simultaneous active links and their transmission duration 126 to keep the required transmit power at a minimum. Furthermore, 127 multihop relaying [14] is capable of conserving the energy of the 128 source node $(\mathrm{SN})$ since intermediate nodes may be employed for 129 reducing the transmission power necessary for maintaining a given 130 end-to-end rate. Hence, we consider the joint optimal design of the 131 transmission rate, transmission power, and scheduling to maximize the 132 NL of energy-constrained WSNs.

133 There is a paucity of contributions in the literature on the issue of 134 cross-layer NL optimization in the context of WSNs. Hoesel et al. 135 [15] proposed a cross-layer approach for jointly optimizing the 136 medium access control (MAC) and routing layer to maximize the 137 NL. Chen and Zhao [8] proposed an efficient MAC protocol that 138 relies both on the channel state information and on the MAC's 139 knowledge of the residual energy to maximize the NL. In [16], 140 Kwon et al. investigated the NL maximization problem of WSNs, 141 which jointly considers the physical layer, the MAC layer, and the 142 routing layer in conjunction with the transmission success probability 143 constraint. Additionally, the tradeoff between NL maximization and 144 application performance was studied in [17] by using cross-layer 145 optimization. A similar study also investigated the tradeoff between 146 the energy consumption and application-layer performance with the 147 aid of cross-layer optimization of WSNs [18]. Another cross-layer ap148 proach conceived for maximizing the NL was proposed in [19], where 149 MAC-aware routing optimization schemes were designed for WSNs 150 that are capable of multichannel access. In [20], Li et al. invoked 151 random linear network coding for the lifetime maximization of wire152 less networks within a fixed-rate system for communicating over both 153 additive white Gaussian noise (AWGN) and Rayleigh fading channels. 154 A different approach to NL maximization was introduced in [21], 155 where both contention and sleep control probabilities of the sensor 156 nodes were utilized for formulating the NL maximization problem, 157 while guaranteeing both the required throughput and the signal-to158 interference-plus-noise ratio (SINR) requirements. Najimi et al. [4] 159 proposed a node selection algorithm for balancing the energy usage of 160 the sensors in a fixed-mode cognitive sensor network. A similar idea

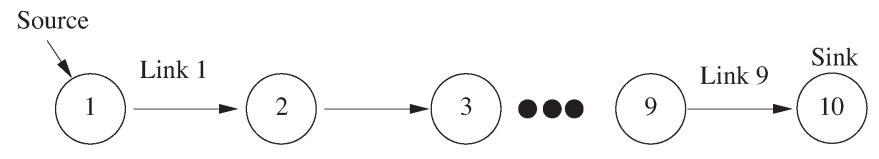

Fig. 1. String topology with $V=10$ nodes, including an SN and a DN.

to that of the optimal control approach invoked for maximizing the NL 161 with the aid of a carefully selected routing probability was exploited 162 in [9], where all the sensors were configured to deplete their energy 163 exactly at the same time for lifetime maximization. Another similar 164 study advocating an effective transmission scheme was proposed in 165 [22], where both the maximum possible energy efficiency and the best 166 possible energy balancing were maintained with the aid of ant colony 167 optimization.

However, all related work aforementioned considers either non- 169 adaptive, i.e., fixed-mode system, or nonfading channel characteristics. 170 An adaptive system conceived for NL maximization was studied by 171 Wang et al. [23], who considered only an interference-free scenario 172 for an AWGN channel by employing the Karush-Kuhn-Tucker (KKT) 173 optimality conditions [24] to the optimal time-division multiple-access 174 (TDMA) NL maximization problem of [12] to derive the analytical 175 expressions of the optimal NL. Madan et al. [12] considered an 176 interference-limited scenario relying on an adaptive system, operating 177 in an AWGN channel, but the impact of the fading channel charac- 178 teristics on the NL was not presented. Wang et al. [23] obtained a 179 closed-form solution for a specific network topology. By contrast, a 180 generalized string network topology consisting of an arbitrary number 181 of nodes is considered in our treatise, where we employ the KKT 182 optimality conditions for obtaining the optimal solution to the NL 183 maximization problem using closed-form expressions. Therefore, we 184 are able to derive analytical expressions of the globally optimal NL for 185 a string network operating in an interference-limited scenario, while 186 communicating either over an AWGN or over fading channels for 187 a given link schedule. Furthermore, the maximum NL, the energy 188 dissipation per node, the average transmission power per link, and the 189 lifetime of all nodes in the network may be obtained. We quantify how 190 the maximum NL decreases as a function of the fading statistics due to 191 the poor channel conditions. Furthermore, it is demonstrated that given 192 a certain network sum rate, the simultaneous scheduling of weakly 193 interfering links benefits from the associated spatial reuse by allowing 194 each node to transmit at a lower rate, which requires a reduced 195 transmission power and hence results in a higher NL. Against this 196 backdrop, the novel contributions of this paper can be summarized as 197 follows.

198

1) The KKT optimality conditions [24] are invoked for deriving 199 the analytical expressions of the globally optimal NL for an 200 interference-limited string topology.

2) In addition to the line-of-sight (LOS) AWGN channel model, 202 the non-LOS Rayleigh block-fading channel model is adopted 203 for studying the effects of fading on the NL.

204

3) The maximum NL is evaluated, and the energy dissipation per 205 node, the average transmission power per link, and the lifetime 206 of all nodes in the network are quantified for a given link 207 schedule and source rate in both LOS AWGN and non-LOS 208 Rayleigh block-fading channels.

4) The substantial effect of the distance among the consecutive 210 nodes on the NL is also analyzed for lower source rates, when 211 operating in a Rayleigh fading channel. The impact of the inter- 212 ferers is also investigated in the context of higher source rates. 213

The remainder of this paper is organized as follows. Section II de- 214 scribes our system model and the constraints of the optimization 215 problem considered. Our problem formulation and solution approach 216 


\begin{tabular}{|c|c|c|c|c|c|c|c|c|c|c|c|c|c|c|c|c|c|c|}
\hline Slots & 1 & 2 & 3 & 4 & 5 & 6 & 7 & 8 & 9 & 10 & 11 & 12 & 13 & 14 & 15 & 16 & 17 & 18 \\
\hline$l_{1-2}$ & • + & & & $\bullet$ & & & - & & & $\stackrel{\bullet}{+}$ & & & - & & & • & & \\
\hline$l_{2-3}$ & & $\cdot$ & & & • & & & $\bullet$ & & & + & & & $\bullet$ & & & $\bullet$ & \\
\hline$l_{3-4}$ & & & • & & & $\bullet$ & & & $\bullet$ & & & $\bullet$ & & & $\bullet$ & & & $\bullet$ \\
\hline$l_{4-5}$ & $\bullet$ & & & • + & & & $\bullet$ & & & • & & & • & & & • & & \\
\hline$l_{5-6}$ & & • & & & + & & & • & & & • & & & $\bullet$ & & & • & \\
\hline$l_{6-7}$ & & & • & & & $\bullet$ & & & • & & & • & & & $\bullet$ & & & • \\
\hline$l_{7-8}$ & $\bullet$ & & & $\bullet$ & & & • + & & & $\bullet$ & & & $\bullet$ & & & • & & \\
\hline$l_{8-9}$ & & • & & & • & & & • & & & • & & & • & & & • & \\
\hline$l_{9-10}$ & & & • & & & $\bullet$ & & & $\bullet$ & & & • & & & • & & & • \\
\hline
\end{tabular}

Fig. 2. Spatially periodic link schedule with time sharing parameter $T=3$ and $T=9$ when $N=18$ and $V=10$.

217 are presented in Section III, and our numerical results are shown in 218 Section IV. Our conclusions are provided in Section V.

\section{SYSTEM MODEL}

220 Here, we first describe the network model, ${ }^{1}$ which relies on a string 221 topology. $^{2}$ Second, we detail our transceiver model in Section II-B, 222 where we evaluate the NL for transmission over both AWGN and 223 block-fading channels. Moreover, our transmission scheduling strategy 224 is also described and exemplified at the end of Section II-B.

\section{A. Network Model}

226 We consider a string topology composed of $V$ sensor nodes, where 227 the SN and the destination node (DN) are linearly connected by 228 intermediate nodes. An example of this string topology for $V=10$ 229 is shown in Fig. 1; hence, the number of links is $L=V-1=9$.

230 Each link is unidirectional, and the antenna of each node is om231 nidirectional. The network can be modeled as a directed graph $\mathcal{G}=$ $232\{\mathcal{V}, \mathcal{L}\}$, where $\mathcal{V}=\{1,2,3, \ldots, V\}$ is the set of all sensor nodes, 233 and $\mathcal{L}=\left\{l_{1,2}, l_{2,3}, \ldots, l_{V-1, V}\right\}$ is the set of all directed links in 234 the network. Here, $l_{i, j}$ represents the directed link spanning from the 235 transmitter node $i$ to receiver node $j$. Therefore, the topology can be 236 modeled with the aid of an incidence matrix of the graph $\mathcal{G}$ given by $237 \mathbf{A} \in \mathbf{R}^{|\mathcal{V}| \times|\mathcal{L}|}$. The entries $a_{v, l}$ of $\mathbf{A}$ are given by

$$
a_{v, l}= \begin{cases}1, & \text { if } v \text { is the transmitter of link } l \\ -1, & \text { if } v \text { is the receiver of link } l \\ 0, & \text { otherwise. }\end{cases}
$$

238 We consider a single commodity flow. Therefore, by the conser239 vation of flow, the constraint $\sum_{l \in \mathcal{O}(v)}\left(\sum_{n=1}^{N} r_{l, n}\right)=\sum_{l \in \mathcal{I}(v)} \times$ $240\left(\sum_{n=1}^{N} r_{l, n}\right)$ may be written for each node $v$ in the absence of an 241 external source or sink, where $N$ is the total number of time slots 242 (TSs) per TDMA frame, and $r_{l, n}$ is the transmission rate of link $l$ in

\footnotetext{
${ }^{1}$ Our network model is a centralized one, where the sink node is assumed to be a control center.

${ }^{2} \mathrm{~A}$ string topology is chosen since, in this simple scenario, the effect of transmission variables on the NL can be explicitly exposed and analyzed. Our string topology scenario is also capable of providing insights concerning a randomly distributed network with many nodes since a specific set of nodes can be assumed to constitute a single route of the randomly distributed network.
}

TS $n$. Additionally, $l \in \mathcal{O}(v)$ denotes the emerging link, and $l \in \mathcal{I}(v) 243$ represents the incoming link of node $v$.

\section{B. Channel Model and MAC Layer Scheme}

In each TS $n$, each node can only act as a transmitter or a receiver. 246 Each transmitter is only allowed to communicate with a single receiver, 247 which cannot receive from other nodes in the same TS. This is due to 248 the half-duplex nature of the transceivers, where nodes communicate 249 on the same shared wireless channel. The channel gain of the link 250 between transmitter $i$ and receiver $j$ is given by $G_{i, j}=1 /\left(d_{i, j}\right)^{m}, 251$ where $d_{i, j}$ is the distance between nodes $i$ and $j$, whereas the path- 252 loss exponent is $m=4$. These channel gains are arranged into a 253 network-channel-gain matrix denoted by $\mathbf{G}$. Each node $v$ is capable 254 of transmitting at a power less than the maximum power of that node 255 denoted by $\left(P_{v}\right)_{\max }$. The total energy dissipation at a node cannot 256 exceed the initial battery energy of that node. No node is allowed to 257 simultaneously transmit multiple data packets, and the link quality is 258 defined by the SINR.

The LOS AWGN channel is modeled by a certain propagation path- 260 loss law and a fixed noise power at the receivers. Given a specific link 261 $l$, the SINR is denoted by $\Gamma_{l}$ in the AWGN channel model. The maxi- 262 mum achievable rate per unit bandwidth is $r_{l}=\log \left(1+K \cdot \Gamma_{l}\right)$ given 263 in nats/s/Hz, where $K=-1.5 / \log (5 \mathrm{BER})$ [25], and BER represents 264 the target bit error ratio (BER) required by the system. Therefore, the 265 SINR is given by [13] $\Gamma_{l_{i, j}, n}=G_{i, j} P_{i, n} /\left(\sum_{i^{\prime} \neq i} G_{i^{\prime}, j} P_{i^{\prime}, n}+N_{0}\right), 266$ where $P_{i, n}$ is the transmission power of node $i$ in TS $n$. Furthermore, 267 $K$ is assumed to incorporate the coding gain and any other gain 268 factors, which is a suitable model for $M$-ary quadrature amplitude 269 modulation (MQAM) associated with $M \geq 4$ [25]. The factor $K$ is 270 assumed absorbed into the gain matrix $\mathbf{G}$.

On the other hand, when considering fading channels, the channel 272 of each link is modeled as a multiplicative Rayleigh fading channel 273 contaminated by the noise added at the receivers. We consider block 274 fading or quasi-static fading, where the fading gain is kept constant 275 throughout the TDMA frame for the link, which represents slowly 276 fading channels, i.e., low Doppler pedestrian speeds. This requires a 277 modification of the SINR used in AWGN channels, which is formu- 278 lated as [25] $\widetilde{\Gamma}_{l_{i, j}, n}=H_{i, j} G_{i, j} P_{i, n} /\left(\sum_{i^{\prime} \neq i} H_{i^{\prime}, j} G_{i^{\prime}, j} P_{i^{\prime}, n}+N_{0}\right), 279$ where $H_{i, j}=\left|h_{i, j}\right|^{2}$ is the fading gain of the link between transmitter 280 $i$ and receiver $j$. 
282 We assume a link scheduling associated with spatially periodic 283 time sharing [12], where we consider a distance $T$ between links 284 that are transmitting in the same TS, and the link is reactivated after 285 every $T$ TSs. Fig. 2 shows the spatially periodic link scheduling for $286 T=3$ and $T=9$. For $T=3$, at the first $T S$, links $l_{1,2}, l_{4,5}, l_{7,8}$ are 287 simultaneously scheduled, and each link is activated six times in total 288 in TSs of $1,4,7,10,13$, and 16. On the other hand, for $T=9$, each TS 289 has only a single active transmission, and each link is activated twice, 290 as shown in Fig. 2.

\section{Problem Formulation AND Solution}

292 Having discussed the assumptions and constraints in Section II, the 293 NL maximization problem [12] can be formulated as in

$$
\begin{aligned}
& \min . \quad z \\
& \text { s.t. } \mathbf{A}\left(\mathbf{r}_{1}+\mathbf{r}_{2}+\cdots+\mathbf{r}_{N}\right)=\mathbf{s} \cdot N \\
& \left(\frac{N_{0}}{G_{i, j}} \cdot e^{r_{l_{i, j}, n}-Q_{l_{i, j}, n}}\right. \\
& \left.+\sum_{l_{i^{\prime}, j^{\prime}} \in \mathcal{L}_{n}, i^{\prime} \neq i} \frac{G_{i^{\prime}, j}}{G_{i, j}} \cdot e^{r_{l_{i, j}, n}+Q_{l_{i^{\prime}, j^{\prime}, n}}-Q_{l_{i, j}, n}}\right) \\
& -1 \leq 0 \quad \forall n, l \in \mathcal{L}_{n} \\
& \sum_{n=1}^{N}\left(\sum_{l \in \mathcal{O}(v) \cap \mathcal{L}_{n}}\left((1+\alpha) \cdot e^{Q_{l_{i, j}, n}}+P_{\mathrm{ct}}\right)\right. \\
& \left.+\sum_{l \in \mathcal{I}(v) \cap \mathcal{L}_{n}} P_{\mathrm{cr}}\right) \leq z \cdot E_{v} \cdot N \quad \forall v \\
& Q_{l_{i, j}, n} \leq \log \left(\left(P_{i}\right)_{\max }\right), l \in \mathcal{L}_{n} \\
& \mathbf{r}_{n} \geq 0 \quad \forall n \\
& r_{l_{i, j}, n}=0 \quad \forall l \notin \mathcal{L}_{n}
\end{aligned}
$$

294 where (4) has been modified, so that it constitutes a strictly convex 295 constraint. See the Nomenclature list on the first page of this paper for 296 the specific parameters utilized in our simulations.

297 The links that are active in TS $n$ are denoted by the set $\mathcal{L}_{n}$, 298 and $\mathbf{s}=\left[s_{1}, 0, \ldots,-s_{1}\right]^{T}$ is the source rate vector, where the first 299 and last elements are nonzero but the remaining elements are set to 300 zero because the first node is the SN and the last node is the DN, 301 and the other nodes act as relay nodes (RN). The variables of the 302 optimization problem are $z, Q_{l, n}$, and $r_{l, n}$, for $l \in \mathcal{L}_{n}, n=1, \ldots, N$. 303 The vector of rate variables associated with TS $n$ is given by $\mathbf{r}_{n}=$ $304\left[r_{l_{1,2}, n}, r_{l_{2,3}, n}, \ldots, r_{l_{V-1, V}, n}\right]^{T}$. Assuming that the transmitter and 305 receiver circuits do not dissipate energy, we can set $P_{\mathrm{ct}}=0$ and $P_{\mathrm{cr}}=0$, 306 where $P_{\mathrm{ct}}$ and $P_{\mathrm{cr}}$ denote the power required by the transmitter 307 and receiver circuits, respectively. Furthermore, we denote the power 308 amplifier inefficiency as $\alpha$ [26]. The lifetime of a node in the network 309 is denoted by $T_{v}$, which corresponds to the time during which the 310 node runs out of battery. The NL is defined as the time during which 311 at least one node completely drains its battery, i.e., we have $T_{\text {net }}=$ $312 \min _{v \neq V, v \in \mathcal{V}} T_{v}$. The objective function $(\mathrm{OF})$ and the constraints of the 313 optimization problem are as follows.

314 1) Objective function-Minimization of reciprocal of the $N L$ : In (2), we minimize $z$ so that the NL is maximized. Here, we used a minimization technique in our problem. We can rewrite (5) as $\sum_{n=1}^{N}\left(\sum_{l \in \mathcal{O}(v) \cap \mathcal{L}_{n}}\left((1+\alpha) \cdot e^{Q_{l_{i, j}, n}}+P_{\mathrm{ct}}\right)+\right.$ $\left.\sum_{l \in \mathcal{I}(v) \cap \mathcal{L}_{n}} P_{\mathrm{cr}}\right) \leq\left(1 / T_{\text {net }}\right) \cdot E_{v} \cdot N$, and we can multiply 318 the left-hand side of the inequality by $T_{\text {net }}$, but the multiplication 319 of the two optimization variables is in general nonconvex. There- 320 fore, we use a change of variable and minimize $z=1 / T_{\text {net }}, 321$ which keeps the right-hand side of the inequality linear and left- 322 hand side convex.

2) Flow conservation constraint: In (3), using matrix A with 324 entries given by 1 ensures that flow conservation is preserved, 325 and physically, this means that the information generated at the 326 $\mathrm{SN}$ has to arrive at the DN.

3) Transmission rate constraint: We have to satisfy the rate con- 328 straint of our interference-limited scenario for each link of the 329 same TS in (4).

4) Energy conservation constraint: Each sensor node can dissipate 331 at most the initial amount of battery energy, which we set to $5000 \mathrm{~J} .332$ Therefore, in (5), the energy conservation constraint is given for 333 each node.

334

5) Transmit power constraint: Equation (6) represents the transmis- 335 sion power at a node, which has to be less than the maximum 336 affordable transmit power of that node.

6) No transmission: Finally, the transmission rate of nodes that are 338 not scheduled for transmission is set to zero in (8).

The optimization problem is solved for the sake of finding the optimal 340 scheme for transmission over each link for a given link schedule, 341 which is defined by the spatially periodic time sharing discussed in 342 Section II-B. However, to obtain the globally optimal solutions, we 343 wish to show that (2)-(8) represent a convex optimization problem, 344 composed of a convex OF, convex inequality constraint functions, and 345 affine equality constraint functions. It is clear that (3) and (8) are affine, 346 (2) and (5)-(7) are convex, and (4) is strictly convex [24]. Therefore, 347 (2)-(8) define a strictly convex optimization problem that has a unique 348 solution. We can convert the problem into its Lagrangian form and 349 rely on the KKT optimality conditions [24] for deriving the analytical 350 expressions of the globally optimal transmission scheme for the string 351 network topology of Fig. 1.

\section{A. Karush-Kuhn-Tucker Optimality Conditions}

Lets us define the sets of the optimization variables and of the 354 Lagrangian multipliers as $\boldsymbol{R}=\left\{r_{l_{1,2}, 1}, \ldots, r_{l_{V-1, V}, N}\right\}, \boldsymbol{Q}=\left\{Q_{l_{1,2}, 1}, 355\right.$ $\left.\ldots, Q_{l_{V-1, V}, N}\right\}, \boldsymbol{\mu}=\left\{\mu_{1}, \ldots, \mu_{V}\right\}, \boldsymbol{\Psi}=\left\{\psi_{l_{1,2}, 1}, \ldots, \psi_{l_{V-1, V}, N}\right\}, 356$ $\boldsymbol{\Omega}=\left\{\omega_{1}, \ldots, \omega_{V-1}\right\}$, and $\boldsymbol{\vartheta}=\left\{\vartheta_{l_{1,2}, 1}, \ldots, \vartheta_{l_{V-1, V}, N}\right\}$. Thus, the 357 partial Lagrangian of (2)-(8) is given by (9), shown at the bottom of 358 the next page, where $\omega_{v}, \psi_{l_{i, j}, n}$, and $\vartheta_{l_{i, j}, n}, \mu_{v}$ are the dual variables 359 associated with the constraints (3)-(6), respectively. Constraints (7) 360 and (8) are taken into account, when deriving the optimal primal 361 variables.

The KKT conditions for (9) are given by (10)-(16), shown at the 363 bottom of the next page, where $\mathcal{I}^{-1}(l)$ denotes the node associated 364 with the incoming link $l$, and $\mathcal{O}^{-1}(l)$ represents the node associated 365 with the outgoing link $l$. Since the primal problem is convex, if 366 $z, \boldsymbol{R}, \boldsymbol{Q}, \boldsymbol{\Omega}, \boldsymbol{\Psi}, \boldsymbol{\vartheta}, \boldsymbol{\mu}$ represent arbitrary points that satisfy the KKT 367 optimality conditions given by the primal feasibility in (3)-(6), the dual 368 feasibility of (16), the complementary slackness in (13)-(15), and the 369 first-order optimality in (10)-(12), then $z, \boldsymbol{R}, \boldsymbol{Q}$ are primal optimal, 370 and $\boldsymbol{\Omega}, \Psi, \vartheta, \boldsymbol{\mu}$ are dual optimal ${ }^{3}$ with zero duality gap ${ }^{4}$ [24].

\footnotetext{
${ }^{3}$ Optimal solution of the primal (original) problem is expressed as primal optimal and the dual problem provides us a lower bound on the optimal value of the original optimization problem. Hence, the dual optimal is a lower bound on the primal optimal.

${ }^{4}$ The duality gap is defined as the difference between the optimal primal and optimal dual solutions.
} 


\section{B. Problem Solution}

373 From (11) and (12), the optimal values of $\boldsymbol{Q}$ and $\boldsymbol{R}$ in iteration $374(t+1)$ are given by (17) and (18), respectively, shown at the bottom of 375 the next page. Note that, due to the interference terms in (11) and (12), 376 each optimal variable in $\boldsymbol{Q}$ and $\boldsymbol{R}$ is dependent on the other variables 377 of $\boldsymbol{Q}$ and $\boldsymbol{R}$, which implies that they are interdependent, hence re378 quiring a centralized solution approach. ${ }^{5}$ Therefore, the Gauss-Seidel

${ }^{5}$ The calculation of both the transmit power and of the rate of a specific node relies on the prior knowledge gleaned from other nodes, possibly from its interferers. Therefore, a control center is required, which handles the variables of the optimization problem and passes the near-instantaneous values of the variables to each of the individual nodes. Compared with a distributed scheme, this centralized solution will impose delay on the system since operations such as channel estimation are required at the initial stage. The near-instantaneous transmission rate and power values computed by the control center constituted by the sink node should be forwarded to each individual node. Therefore, a nonnegligible delay will be imposed on the reception of the sink node. algorithm [27] is utilized for iteratively updating these variables in a 379 circular fashion.

The dual $\mathrm{OF}$ is defined as the minimum value of the Lagrangian 381 (9) over $z, \boldsymbol{R}, \boldsymbol{Q}$ given by $g(\boldsymbol{\Omega}, \boldsymbol{\Psi}, \boldsymbol{\vartheta}, \boldsymbol{\mu})=\inf _{z, \boldsymbol{Q}} \mathcal{L}(z, \boldsymbol{R}, \boldsymbol{Q}, \boldsymbol{\Omega}, \boldsymbol{\Psi}, 382$ $\boldsymbol{\vartheta}, \boldsymbol{\mu})$, which is a linear problem even if the primal problem is 383 nonconvex. The dual function $g(\boldsymbol{\Omega}, \boldsymbol{\Psi}, \boldsymbol{\vartheta}, \boldsymbol{\mu})$ may be maximized to 384 find a lower bound for the optimal value of the primal problem. Then, 385 we can write the dual problem as follows:

$$
\begin{aligned}
\max _{\boldsymbol{\Omega}, \boldsymbol{\Psi}, \boldsymbol{\vartheta}, \boldsymbol{\mu}} & g(\boldsymbol{\Omega}, \boldsymbol{\Psi}, \boldsymbol{\vartheta}, \boldsymbol{\mu}) \\
\text { s.t. } & \boldsymbol{\Omega} \geq 0, \boldsymbol{\Psi} \geq 0, \boldsymbol{\vartheta} \geq 0
\end{aligned}
$$

which is a linear optimization problem. When the primal problem is 387 convex, this lower bound is tight; therefore, the duality gap is zero. 388 Since the dual problem is continuously differentiable, the gradient 389 ascent algorithm [27] is utilized to solve the maximization problem 390 by simply evaluating a series of closed-form expressions. The gradient 391

$$
\begin{aligned}
& \mathcal{L}(z, \boldsymbol{R}, \boldsymbol{Q}, \boldsymbol{\Omega}, \boldsymbol{\Psi}, \boldsymbol{\vartheta}, \boldsymbol{\mu})=z+\sum_{v=1}^{V-1} \omega_{v} \cdot\left[\sum_{n=1}^{N}\left(\sum_{l \in \mathcal{O}(v) \cap \mathcal{L}_{n}}\left((1+\alpha) \cdot e^{Q_{l_{i, j}, n}}\right)\right)-z \cdot E_{v} \cdot N\right] \\
& +\sum_{n=1}^{N} \sum_{l \in \mathcal{L}_{n}} \psi_{l_{i, j}, n} \cdot\left[\left(\frac{N_{0}}{G_{i, j}} e^{r_{l_{i, j}, n}-Q_{l_{i, j}, n}}+\sum_{l_{i^{\prime}, j^{\prime}} \in \mathcal{L}_{n}, i^{\prime} \neq i} \frac{G_{i^{\prime}, j}}{G_{i, j}} e^{r_{l_{i, j}, n}+Q_{l_{i^{\prime}, j^{\prime}, n}}-Q_{l_{i, j}, n}}\right]-1\right] \\
& +\sum_{n=1}^{N} \sum_{l \in \mathcal{L}_{n}} \vartheta_{l_{i, j}, n} \cdot\left[Q_{l_{i, j}, n}-\log \left(\left(P_{i}\right)_{\max }\right)\right]+\sum_{v=1}^{V} \mu_{v} \cdot\left[\sum_{l \in \mathcal{I}(v)}\left(\sum_{n=1}^{N} r_{l_{i, j}, n}\right)-\sum_{l \in \mathcal{O}(v)}\left(\sum_{n=1}^{N} r_{l_{i, j}, n}\right)\right]
\end{aligned}
$$

$$
\begin{aligned}
& \frac{\partial \mathcal{L}}{\partial z}=1-\sum_{v=1}^{V} \omega_{v}\left(E_{v} \cdot N\right)=0 \\
& \frac{\partial \mathcal{L}}{\partial r_{l_{i, j}, n}}=\mu_{\mathcal{I}^{-1}(l)}-\mu_{\mathcal{O}^{-1}(l)}+\psi_{l_{i, j}, n}\left(\frac{N_{0}}{G_{i, j}} e^{r_{l_{i, j}, n}-Q_{l_{i, j}, n}}+\sum_{l_{i^{\prime}, j^{\prime}} \in \mathcal{L}_{n}, i^{\prime} \neq i} \frac{G_{i^{\prime}, j}}{G_{i, j}} e^{r_{l_{i, j}, n}+Q_{l_{i^{\prime}, j^{\prime}, n}}-Q_{l_{i, j}, n}}\right)=0 \quad \forall l, n \\
& \frac{\partial \mathcal{L}}{\partial Q_{l_{i, j}, n}}=\omega_{\mathcal{O}^{-1}(l)}\left((1+\alpha) e^{Q_{l_{i, j}, n}}\right)+\vartheta_{l_{i, j}, n} \\
& -\psi_{l_{i, j}, n}\left(\frac{N_{0}}{G_{i, j}} e^{r_{l_{i, j}, n}-Q_{l_{i, j}, n}}+\sum_{l_{i^{\prime}, j^{\prime}} \in \mathcal{L}_{n}, i^{\prime} \neq i} \frac{G_{i^{\prime}, j}}{G_{i, j}} e^{r_{l_{i, j}, n}+Q_{l_{i^{\prime}, j^{\prime}, n}}-Q_{l_{i, j}, n}}\right)=0 \quad \forall l, n \\
& 0=\sum_{v=1}^{V-1} \omega_{v} \cdot\left[\sum_{n=1}^{N}\left(\sum_{l \in \mathcal{O}(v) \cap \mathcal{L}_{n}}\left((1+\alpha) e^{Q_{l_{i, j}, n}}\right)\right)-z \cdot E_{v} \cdot N\right] \\
& 0=\psi_{l_{i, j}, n} \cdot\left[\left(\frac{N_{0}}{G_{i, j}} e^{r_{l_{i, j}, n}-Q_{l_{i, j}, n}}+\sum_{l_{i^{\prime}, j^{\prime}} \in \mathcal{L}_{n}, i^{\prime} \neq i} \frac{G_{i^{\prime}, j}}{G_{i, j}} e^{r_{l_{i, j}, n}+Q_{l_{i^{\prime}, j^{\prime}, n}}-Q_{l_{i, j}, n}}\right)-1\right] \forall l, n \\
& 0=\vartheta_{l_{i, j}, n} \cdot\left[Q_{l_{i, j}, n}-\log \left(\left(P_{i}\right)_{\max }\right)\right] \quad \forall l, n \\
& \omega_{v} \geq 0, \psi_{l_{i, j}, n} \geq 0, \vartheta_{l_{i, j}, n} \geq 0
\end{aligned}
$$


392 of the Lagrangian function defines the search directions at the current 393 point. Each dual variable is incremented in the direction of the positive 394 gradient in (19)-(22), shown at the bottom of the page, where $t$ is the 395 iteration index, and $[\cdot]^{+}$denotes $\max (0, \cdot)$. Provided that $\Delta_{\Omega}>0$, $396 \Delta_{\Psi}>0, \Delta_{\vartheta}>0$, and $\Delta_{\mu}>0$ are sufficiently small positive step 397 sizes, the dual variables $\boldsymbol{\Omega}^{t}, \boldsymbol{\Psi}^{t}, \boldsymbol{\vartheta}^{t}$, and $\boldsymbol{\mu}^{t}$ converge to the dual 398 optimal variables $\boldsymbol{\Omega}^{*}, \Psi^{*}, \boldsymbol{\vartheta}^{*}$, and $\boldsymbol{\mu}^{*}$, respectively, as $t \rightarrow \infty$. In 399 our case, the optimization problem shown in (2)-(8) is strictly convex; 400 thus, the duality gap is zero, and the solution is unique.

\section{1 \\ IV. EXPERIMENTAL RESULTS}

402 In our experiments, we use the parameters of $d=1 \mathrm{~m}, \alpha=0.01$ 403 [26], $K=1, N_{0}=1 \mathrm{dBm} / \mathrm{Hz}, E_{v}=5000 \mathrm{~J}^{6},\left(P_{i}\right)_{\max }=50 \mathrm{~W}, N=$ $40418, s_{1}=\{0.2,0.3,0.4,0.5,0.6,0.7\}$ nats $/ \mathrm{s} / \mathrm{Hz} \approx\{0.29,0.43,0.58$, $4050.72,0.87,1.01\}$ bits/s/Hz, $T=\{3,4,5,6,7,8,9\}$, convergence toler406 ance of iterative algorithm $\epsilon=10^{-5}$.

407 Fig. 3 shows the NL versus source rate trends for a fixed link 408 schedule and for various spatially periodic time sharing parameters $409 T$, where the channel in each link is a LOS AWGN channel char410 acterized by fixed noise power. As expected, the NL decays as a 411 function of the source rate, as shown in Fig. 3. This is because a 412 higher source rate requires a higher transmission rate and, hence, 413 higher transmission power. Furthermore, in our model, the weakly 414 interfering nodes are scheduled to transmit simultaneously; hence,

${ }^{6}$ For example, this is the energy storage capacity of an AAA alkaline longlife battery.

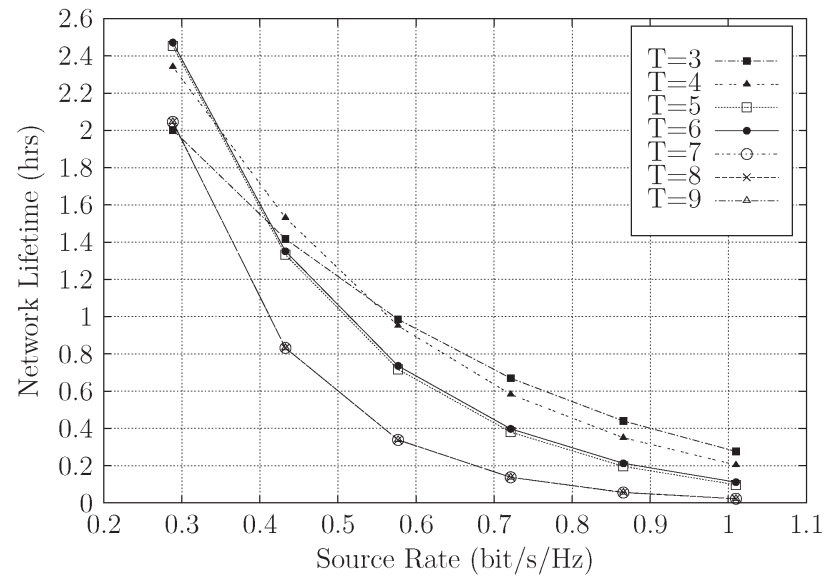

Fig. 3. Network lifetime for different spatially periodic schedules and source rates in the AWGN channel.

each link becomes capable of transmitting at a lower rate, while still 415 satisfying all the transmit requirements of the SN. This necessitates 416 lower transmission power. Using the $T=9$ spatially periodic time 417 schedule of Fig. 2 corresponds to a TDMA scheme since there is only 418 a single transmission in each TS, as shown in Fig. 2. However, since 419 the time frame of Fig. 2 consists of 18 TSs, a specific link is scheduled 420 to transmit twice during the whole time frame. Despite the fact that the 421 $T=9$ link schedule does not impose any interference, it results in the 422 lowest NL according to Fig. 3. Although interference is present in 423

$$
\begin{aligned}
& Q_{l_{i, j}, n}^{t+1}=\log \left[( \mu _ { i } ^ { t } - \mu _ { j } ^ { t } - \vartheta _ { l _ { i , j } , n } ^ { t } ) \cdot \left(\omega_{i}^{t}(1+\alpha)+\sum_{l_{i^{\prime}, j^{\prime}} \in \mathcal{L}_{n}, l_{i^{\prime}, j^{\prime}} \neq l_{i, j}, i^{\prime} \geq i} \psi_{l_{i^{\prime}, j^{\prime}}, n}^{t}\left(\frac{G_{i, j^{\prime}}}{G_{i^{\prime}, j^{\prime}}} \cdot e^{r_{l_{i^{\prime}, j^{\prime}}, n}-Q_{l_{i^{\prime}, j^{\prime}, n}^{t}}}\right)\right.\right. \\
& \left.\left.+\sum_{l_{i^{\prime}, j^{\prime}} \in \mathcal{L}_{n}, l_{i^{\prime}, j^{\prime}} \neq l_{i, j}, i^{\prime}<i} \psi_{l_{i^{\prime}, j^{\prime}}, n}^{t}\left(\frac{G_{i, j^{\prime}}}{G_{i^{\prime}, j^{\prime}}} \cdot e^{r_{l_{i^{\prime}, j^{\prime}, n}^{t}}-Q_{l_{i^{\prime}, j^{\prime}}}^{t+1}}\right)\right)^{-1}\right] \forall l, n \\
& r_{l_{i, j}, n}^{t+1}=\log \left[\frac{\mu_{i}^{t}-\mu_{j}^{t}}{\psi_{l_{i, j}, n}^{t} \cdot\left(\frac{N_{0}}{G_{i, j}}+\sum_{l_{i^{\prime}, j^{\prime}} \in \mathcal{L}_{n}, l_{i^{\prime}, j^{\prime}} \neq l_{i, j}} \frac{G_{i^{\prime}, j}}{G_{i, j}} \cdot e^{Q_{l_{i^{\prime}, j^{\prime}, n}}^{t+1}}\right)}\right]+Q_{l_{i, j}, n}^{t+1} \quad \forall l, n
\end{aligned}
$$

$$
\begin{aligned}
& \omega^{t+1}=\left[\omega^{t}+\Delta_{\omega}\left(\sum_{n=1}^{N}\left(\sum_{l \in \mathcal{O}(v) \cap \mathcal{L}_{n}}\left((1+\alpha) \cdot e^{Q_{l_{i, j}, n}}\right)\right)-z \cdot E_{v} \cdot N\right)\right]^{+} \\
& \psi^{t+1}=\left[\psi^{t}+\Delta_{\psi}\left(\left(\frac{N_{0}}{G_{i, j}} e^{r_{l_{i, j}, n}-Q_{l_{i, j}, n}}+\sum_{l_{i^{\prime}, j^{\prime}} \in \mathcal{L}_{n}, i^{\prime} \neq i} \frac{G_{i^{\prime}, j}}{G_{i, j}} e^{r_{l_{i, j}, n}+Q_{l_{i^{\prime}, j^{\prime}, n}}-Q_{l_{i, j}, n}}\right)-1\right)\right]^{+} \\
& \vartheta^{t+1}=\left[\vartheta^{t}+\Delta_{\vartheta}\left(Q_{l_{i, j}, n}-\log \left(\left(P_{i}\right)_{\max }\right)\right)\right]^{+} \\
& \mu^{t+1}=\left[\mu^{t}+\Delta_{\mu}\left(\sum_{l \in \mathcal{I}(v)}\left(\sum_{n=1}^{N} r_{l_{i, j}, n}\right)-\sum_{l \in \mathcal{O}(v)}\left(\sum_{n=1}^{N} r_{l_{i, j}, n}\right)\right)\right]
\end{aligned}
$$




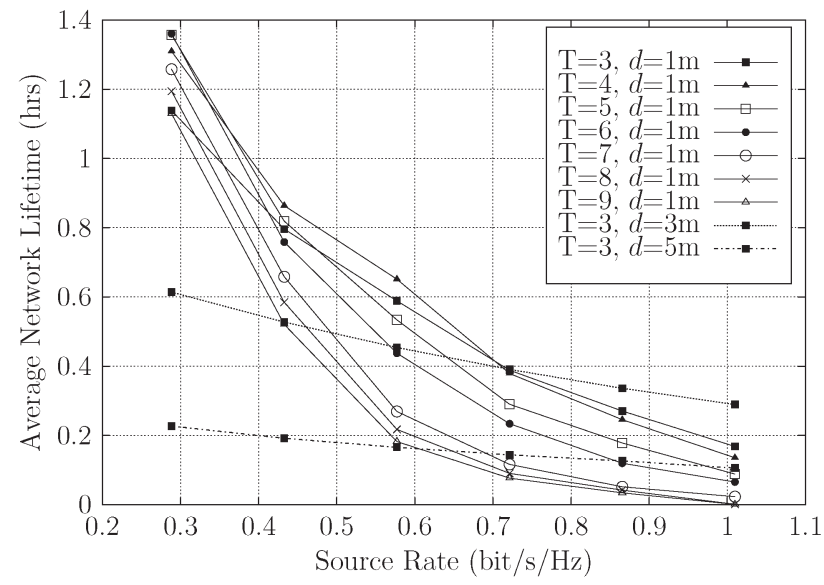

Fig. 4. Network lifetime for different spatially periodic schedules and source rates in a block-fading channel.

424 the $T=3$ scenario since each link can be activated three times more 425 often than in the $T=9$ scenario, each link in the $T=3$ scenario can 426 be activated at lower transmission power, while still satisfying the end427 to-end rate constraint. Therefore, the spatial reuse assisted us in the $428 T=3$ scenario for increasing the NL. We can follow Fig. 2 to find out 429 how many transmissions there are per link for a given value of $T$. For 430 example, the $T=3$ schedule allows a link to be scheduled six times, 431 which requires a reduced transmission rate, since the total source rate 432 that is delivered over different TSs is using six transmissions. From the 433 flow conservation equality constraint of the optimization problem seen 434 in (3), we have $\mathbf{A}\left(\mathbf{r}_{1}+\mathbf{r}_{2}+\cdots+\mathbf{r}_{N}\right)=\mathbf{s} \cdot N$. For example, let us 435 assume that the source rate equals to $0.29 \mathrm{bits} / \mathrm{s} / \mathrm{Hz}$. Then, we obtain $43618 \cdot 0.29=5.22 \mathrm{bits} / \mathrm{s} / \mathrm{Hz}$, which has to be divided into six transmis437 sions, corresponding to a $0.87 \mathrm{bits} / \mathrm{s} / \mathrm{Hz}$ per link transmission rate for $438 T=3$. However, when we have $T=9$, we obtain a $2.61-\mathrm{bits} / \mathrm{s} / \mathrm{Hz}$ 439 per link transmission rate since a link is only activated twice during the 440 whole time frame. Therefore, the transmission rate per link converges 441 to $0.87 \mathrm{bits} / \mathrm{s} / \mathrm{Hz}$ for $T=3$ and $2.61 \mathrm{bits} / \mathrm{s} / \mathrm{Hz}$ for $T=9$. Hence, $442 T=9$ requires three times as much transmission power as $T=3$. 443 The required transmit power in weakly interfering links is quite low 444 compared with that for $T=9$, which is the scenario requiring the 445 highest transmission rate. Hence, again, we surmise that simultaneous 446 scheduling benefits from reduced transmission power due to its re447 duced transmission rate per link. This is because the spatially periodic 448 schedule allows us to schedule more transmissions during the same 449 TS or to activate the same link more than once in different TSs. This 450 explains the steep decay of the NL for $T=9$.

451 When considering the effects of node density on a given fixed link 452 schedule, we expect a network supporting less than $V=10$ nodes to 453 be exposed to less interference. Therefore, the transmission power of 454 each link can be reduced without reducing the end-to-end transmission 455 rate, which results in a higher NL. On the other hand, upon increasing 456 the node density, we expect the NL to decrease since more interferers 457 are introduced, but the same transmission rate is required.

458 Fig. 4 represents the NL versus source rate tradeoff for a fixed 459 link schedule and for various spatially periodic time sharing parameter 460 values of $T$ when each link obeys an independent and identically dis461 tributed Rayleigh block-fading channel. Naturally, the NL was reduced 462 compared with the results of Fig. 3 recorded for an AWGN channel 463 due to requiring higher transmit power to combat the effects of fading. 464 We also analyzed the impact of the internode distance on the NL for 465 the $T=3$-based link schedule, when communicating over a Rayleigh 466 fading channel, as shown in Fig. 4. Increasing the distance between 467 the consecutive nodes substantially reduced the NL, particularly for

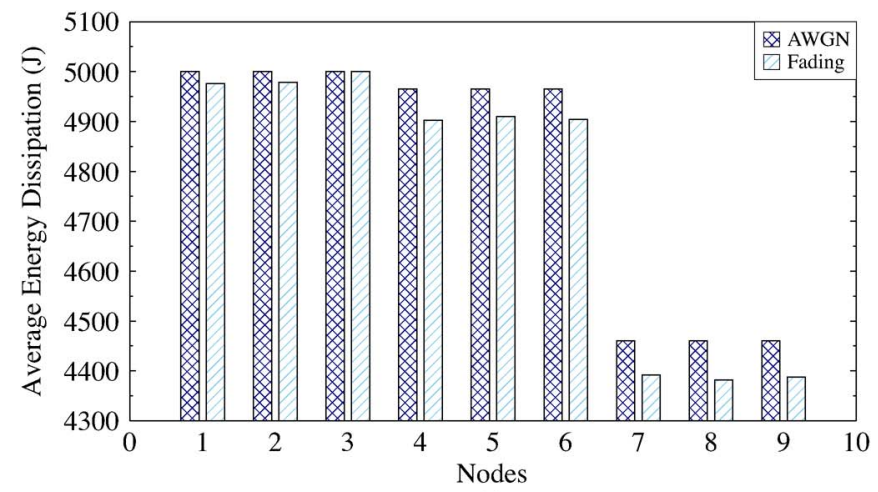

(a)

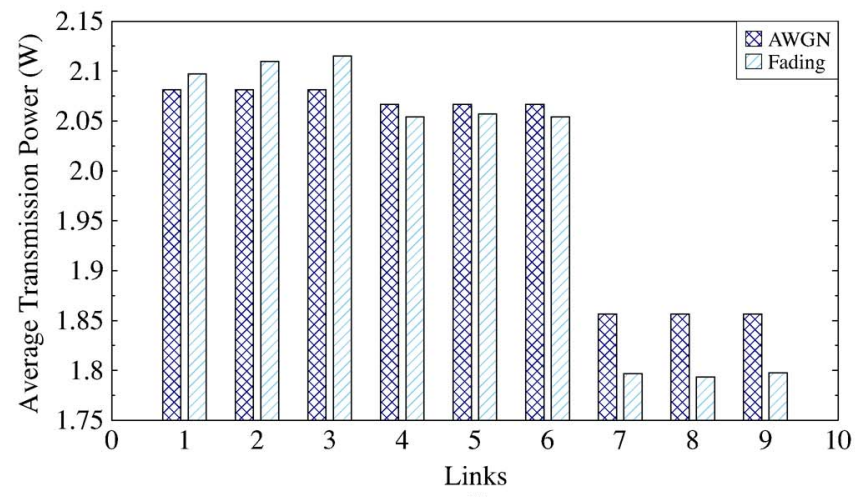

(b)

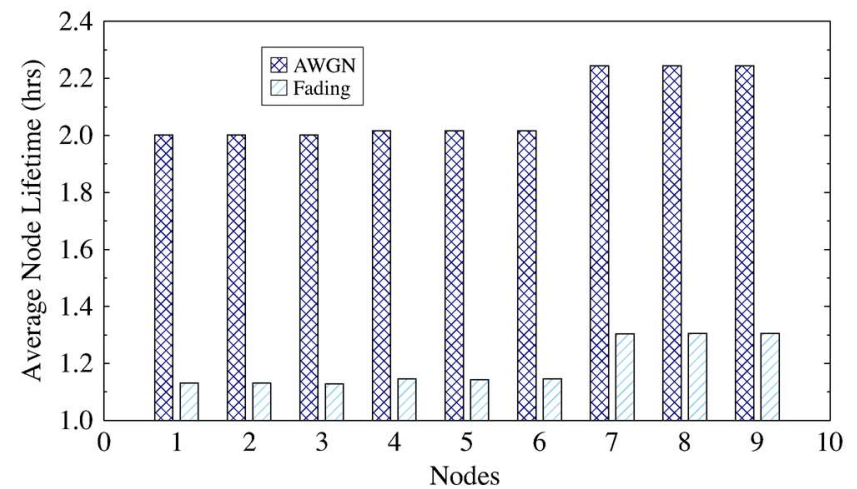

(c)

Fig. 5. Energy dissipation per node, average transmit power per link and lifetime of all nodes in the network in both AWGN and fading channels for the $T=3$ link schedule at a source rate of $0.29 \mathrm{bits} / \mathrm{s} / \mathrm{Hz}$. (a) Energy dissipation per node. (b) Average transmit power per link. (c) Lifetime of all nodes in the network.

lower source rates. However, quite surprisingly, increasing the distance 468 between the consecutive nodes from 1 to $3 \mathrm{~m}$ resulted in an improved 469 NL for higher source rates. This is due to the reduced impact of 470 the interferers located at a higher distance. More explicitly, although 471 the transmit power required had to be increased to satisfy the rate 472 constraint, at the same time the interferers were moved a bit further 473 away. Therefore, the total energy dissipation of the $d=3 \mathrm{~m}$ scenario 474 is still lower than that of the $d=1 \mathrm{~m}$ scenario associated with higher 475 source rates.

476

Furthermore, we comprehensively study the energy dissipation per 477 node, the average transmission power per link, and the lifetime of all 478 sensor nodes in the network. Fig. 5 shows the energy dissipation per 479 node, the average transmission power per link, and the lifetime of all 480 nodes in the network in both AWGN and fading channels for the $T=3481$ link schedule of Fig. 2 at a source rate of 0.29 bits/s/Hz. In the network 482 
483 topology considered, the transmissions from the first three nodes suffer 484 from the highest amount of interference. This is because their receiving 485 nodes are closer to their potential interferers, when compared with 486 any other sets of nodes. Therefore, to satisfy the flow conservation 487 constraints, these nodes must transmit at higher power, as shown in 488 Fig. 5(b). Thus, in an AWGN channel, the first three nodes in the 489 network dissipate their 5000-J initial amount of energy faster than the 490 other nodes since the energy dissipation is proportional to the transmit 491 power, as shown in Fig. 5(a), whereas in the Rayleigh block-fading 492 channel, the third node runs out of battery first, which also determines 493 the lifetime of the WSNs. The required transmit power of the third 494 link is higher than that in the AWGN channel scenario. This increase 495 in transmit power is required to overcome the effect of fading.

496 The average transmit power per link is calculated by summing the 497 transmit power values per link and then by dividing it by the number of 498 TSs that the same link was allowed to transmit. For the first three links 499 operating in the AWGN channel, the required transmission power per 500 link is higher than that of the rest of the links. Since requiring a high 501 transmit power results in dissipating more energy, the lifetime of those 502 nodes is reduced, as shown in Fig. 5(c).

503 Upon comparing the AWGN and fading channel scenarios in Fig. 5, 504 we observe that they follow a similar trend. An observation is that 505 the average transmit power per link of the seventh, eighth, and ninth 506 nodes in Fig. 5(b) is slightly lower for the fading channel than for the 507 AWGN channel. However, interestingly, the average transmit power 508 per link of the third node in Fig. 5(b) recorded for the fading channel 509 is slightly higher than that of the AWGN channel. Therefore, the need 510 for a high transmit power necessitates higher energy dissipation for 511 that particular node. Hence, the NL is reduced, which can also be 512 observed by comparing Figs. 3 and 4. Fig. 3 shows that the NL of 513 the WSN in the AWGN channel recorded for the $T=3$ link schedule 514 and for 0.29 bits/s/Hz source rate is approximately $2 \mathrm{~h}$. By contrast, 515 Fig. 4 shows that the NL of the WSN operating in a Rayleigh fading 516 scenario for the $T=3$ link schedule and for 0.29 -bits $/ \mathrm{s} / \mathrm{Hz}$ source rate 517 indicates approximately an NL of $1.13 \mathrm{~h}$. This earlier node failure of 518 the fading scenario is due to the poor channel conditions, where the 519 fading required higher transmit power in the third node, as shown in 520 Fig. 5(b). Therefore, this earlier node failure shortened the NL of the 521 WSNs in fading channels.

522 To put the given results into context, we apply our analysis to the 523 environmental sensor networks of [28], where the relation between 524 glaciers and climate change was studied. In their work, Martinez et al. 525 [28] transmit data only once per day for a 0.5 -s time slot. In this 526 specific application and considering our results in Fig. 5 for $T=3$ and 527 a source rate of $0.29 \mathrm{bits} / \mathrm{s} / \mathrm{Hz}$, the battery will serve communications 528 for $7200 \mathrm{~s}$, which means that the NL will be around four years and 529 three months in the LOS AWGN channel. We also consider what NL 530 we can achieve if the environmental conditions are more challenging 531 and the channel is exposed to the severe environments mentioned in 532 [28], which may be modeled by a non-LOS Rayleigh block-fading 533 channel. Activating the communication channel once per day in fading 534 conditions will lead us to an NL of around two years and six months.

\section{CONCLUSION}

536 We evaluated the optimal NL in an interference-limited scenario for 537 an optimal transmit rate and power, when considering the so-called 538 spatially periodic time sharing scheme of Fig. 2. The maximization 539 of NL was formulated as a nonlinear optimization problem taking into 540 account the link scheduling, the transmission rates, and transmit power 541 of all active TSs. The original nonlinear problem was converted into 542 a convex optimization problem by employing an approximation of the 543 SINR. We then derived the Lagrangian form of the convex optimiza- tion problem and employed the KKT optimality conditions [24] for 544 deriving analytical expressions of the globally optimal transmit rate 545 and power for our specific network topology. Finally, we obtained the 546 maximum NL for both AWGN and Rayleigh fading channels. Our 547 numerical results illustrated that fading has a detrimental impact on 548 the achievable NL due to the poor channel conditions that require an 549 increased transmit power to combat the effects of the fading. Further- 550 more, the simultaneous scheduling of links that interfere only weakly 551 allowed us to take advantage of spatial reuse, where the activation 552 of simultaneous transmissions at reduced rates necessitates reduced 553 transmission power, which results in extending the NL. From this 554 paper, we can conclude that the choice of scheduling depends on the 555 application since a lower source rate favors infrequent transmissions 556 requiring low transmit power, which do not suffer from interference, 557 when aiming for extending the NL. However, for higher source rates, 558 a higher NL can be achieved by aggressive spatial reuse.

Given the limitations of the centralized solution approach men- 560 tioned in Section III-B, the focus of this paper is on the information 561 delay analysis. We also plan to extend our string topology model to a 562 random network topology, where a single string ( $\mathrm{SN}-\mathrm{DN}$ pair) can be 563 assumed to constitute a single route of the random topology. Nonethe- 564 less, conceiving distributed solutions for avoiding the limitations of our 565 centralized scheme constitutes attractive future research directions. 566

REFERENCES

[1] I. Dietrich and F. Dressler, "On the lifetime of wireless sensor networks," 568 ACM Trans. Sensor Netw., vol. 5, no. 1, pp. 5:1-5:39, Feb. 2009.

[2] W. Liu, K. Lu, J. Wang, G. Xing, and L. Huang, "Performance analysis of 570 wireless sensor networks with mobile sinks," IEEE Trans. Veh. Technol., 571 vol. 61, no. 6, pp. 2777-2788, Jul. 2012.

572

[3] J. Chen, J. Li, and T. Lai, "Trapping mobile targets in wireless sensor 573 networks: An energy-efficient perspective," IEEE Trans. Veh. Technol., 574 vol. 62, no. 7, pp. 3287-3300, Sep. 2013.

[4] M. Najimi, A. Ebrahimzadeh, S. Andargoli, and A. Fallahi, "Lifetime 576 maximization in cognitive sensor networks based on the node selection," 577 IEEE Sensors J., vol. 14, no. 7, pp. 2376-2383, Jul. 2014.

[5] J. Du, K. Wang, H. Liu, and D. Guo, "Maximizing the lifetime of 579 k-discrete barrier coverage using mobile sensors," IEEE Sensors J., 580 vol. 13, no. 12, pp. 4690-4701, Dec. 2013.

[6] H. Salarian, K. Chin, and F. Naghdy, "An energy-efficient mobile-sink 582 path selection strategy for wireless sensor networks," IEEE Trans. Veh. 583 Technol., vol. 63, no. 5, pp. 2407-2419, Jun. 2014.

[7] J. W. Jung and M. Weitnauer, "On using cooperative routing for lifetime 585 optimization of multi-hop wireless sensor networks: Analysis and guide- 586 lines," IEEE Trans. Commun., vol. 61, no. 8, pp. 3413-3423, Aug. 2013. 587

[8] Y. Chen and Q. Zhao, "On the lifetime of wireless sensor networks," IEEE 588 Commun. Lett., vol. 9, no. 11, pp. 976-978, Nov. 2005.

[9] C. Cassandras, T. Wang, and S. Pourazarm, "Optimal routing and en- 590 ergy allocation for lifetime maximization of wireless sensor networks 591 with nonideal batteries," IEEE Trans. Control Netw. Syst., vol. 1, no. 1, 592 pp. 86-98, Mar. 2014.

[10] D. Yuan, V. Angelakis, L. Chen, E. Karipidis, and E. Larsson, "On opti- 594 mal link activation with interference cancelation in wireless networking," 595 IEEE Trans. Veh. Technol., vol. 62, no. 2, pp. 939-945, Feb. 2013.

[11] Z. Yang, Q. Zhang, and Z. Niu, "Throughput improvement by joint relay 597 selection and link scheduling in relay-assisted cellular networks," IEEE 598 Trans. Veh. Technol., vol. 61, no. 6, pp. 2824-2835, Jul. 2012.

599

[12] R. Madan, S. Cui, S. Lall, and A. Goldsmith, "Cross-layer design for 600 lifetime maximization in interference-limited wireless sensor networks," 601 IEEE Trans. Wireless Commun., vol. 5, no. 11, pp. 3142-3152, Nov. 2006. 602

[13] A. Goldsmith, Wireless Communications. Cambridge, U.K.: Cambridge 603 Univ. Press, 2005.

[14] H. Yetgin, K. T. K. Cheung, and L. Hanzo, "Multi-objective routing 605 optimization using evolutionary algorithms," in Proc. IEEE WCNC, Paris, 606 France, Apr. 2012, pp. 3030-3034.

[15] L. Van Hoesel, T. Nieberg, J. Wu, and P. J. M. Havinga, "Prolonging 608 the lifetime of wireless sensor networks by cross-layer interaction," IEEE 609 Wireless Commun. Mag., vol. 11, no. 6, pp. 78-86, Dec. 2004.

610

[16] H. Kwon, T. H. Kim, S. Choi, and B. G. Lee, "A cross-layer strategy 611 for energy-efficient reliable delivery in wireless sensor networks," IEEE 612 Trans. Wireless Commun., vol. 5, no. 12, pp. 3689-3699, Dec. 2006. 
614 [17] H. Nama, M. Chiang, and N. Mandayam, "Utility-lifetime trade-off in self-regulating wireless sensor networks: A cross-layer design approach," in Proc. IEEE ICC, Istanbul, Turkey, Jun. 2006, vol. 8, pp. 3511-3516.

617 [18] J. Zhu, S. Chen, B. Bensaou, and K.-L. Hung, "Tradeoff between life618 time and rate allocation in wireless sensor networks: A cross layer 619 approach," in Proc. 26th IEEE INFOCOM, Anchorage, AK, USA, 620 May 2007, pp. 267-275.

621 [19] S. Ehsan, B. Hamdaoui, and M. Guizani, "Radio and medium access 622 contention aware routing for lifetime maximization in multichannel sensor 623 networks," IEEE Trans. Wireless Commun., vol. 11, no. 9, pp. 3058-3067, 624 Sep. 2012

625 [20] P. Li, S. Guo, and V. Leung, "Maximum-lifetime coding tree for multicast 626 in lossy wireless networks," IEEE Wireless Commun. Lett., vol. 2, no. 3 , pp. 295-298, Jun. 2013.

21] J.-H. Jeon, H.-J. Byun, and J.-T. Lim, "Joint contention and sleep control for lifetime maximization in wireless sensor networks," IEEE Commun. Lett., vol. 17, no. 2, pp. 269-272, Feb. 2013.
[22] X. Liu, "A transmission scheme for wireless sensor networks using ant 631 colony optimization with unconventional characteristics," IEEE Commun. 632 Lett., vol. 18, no. 7, pp. 1214-1217, Jul. 2014.

[23] H. Wang, N. Agoulmine, M. Ma, and Y. Jin, "Network lifetime optimiza- 634 tion in wireless sensor networks," IEEE J. Sel. Areas Commun., vol. 28, 635 no. 7, pp. 1127-1137, Sep. 2010.

[24] S. P. Boyd and L. Vandenberghe, Convex Optimization. Cambridge, 637 U.K.: Cambridge Univ. Press, 2004.

[25] A. Goldsmith and S.-G. Chua, "Variable-rate variable-power MQAM for 639 fading channels," IEEE Trans. Commun., vol. 45, no. 10, pp. 1218-1230, 640 Oct. 1997.

641

[26] M. Albulet, RF Power Amplifiers. Raleigh, NC, USA: SciTech, 2001. 642

[27] D. Palomar and M. Chiang, "A tutorial on decomposition methods for 643 network utility maximization," IEEE J. Sel. Areas Commun., vol. 24, 644 no. 8, pp. 1439-1451, Aug. 2006.

[28] K. Martinez, J. Hart, and R. Ong, "Environmental sensor networks," 646 Computer, vol. 37, no. 8, pp. 50-56, Aug. 2004. 


\section{AUTHOR QUERIES}

AUTHOR PLEASE ANSWER ALL QUERIES

AQ1 = Please provide keywords.

END OF ALL QUERIES 


\section{Correspondence}

\section{Cross-Layer Network Lifetime Maximization in Interference-Limited WSNs}

\author{
Halil Yetgin, Student Member, IEEE, \\ Kent Tsz Kan Cheung, Student Member, IEEE, \\ Mohammed El-Hajjar, Member, IEEE, and \\ Lajos Hanzo, Fellow, IEEE
}

7 Abstract-In wireless sensor networks (WSNs), the network lifetime 8 (NL) is a crucial metric since the sensor nodes usually rely on lim9 ited energy supply. In this paper, we consider the joint optimal design 10 of the physical, medium access control (MAC), and network layers to 11 maximize the NL of the energy-constrained WSN. The problem of NL 12 maximization can be formulated as a nonlinear optimization problem 13 encompassing the routing flow, link scheduling, transmission rate, and 14 power allocation operations for all active time slots (TSs). The resultant 15 nonconvex rate constraint is relaxed by employing an approximation of 16 the signal-to-interference-plus-noise ratio (SINR), which transforms the 17 problem to a convex one. Hence, the resultant dual problem may be 18 solved to obtain the optimal solution to the relaxed problem with a zero 19 duality gap. Therefore, the problem is formulated in its Lagrangian form, 20 and the Karush-Kuhn-Tucker (KKT) optimality conditions are employed 21 for deriving analytical expressions of the globally optimal transmission 22 rate and power allocation variables for the network topology considered.

23 The nonlinear Gauss-Seidel algorithm is adopted for iteratively updating

24 the rate and power allocation variables using these expressions until 25 convergence is attained. Furthermore, the gradient method is applied for 26 updating the dual variables in each iteration. Using this approach, the 27 maximum NL, the energy dissipation per node, the average transmission 28 power per link, and the lifetime of all nodes in the network are evaluated 29 for a given source rate and fixed link schedule under different channel 30 conditions.

31 Index Terms-Author, please supply index terms/keywords for your 32 paper. To download the IEEE Taxonomy go to http://www.ieee.org/ 33 documents/taxonomy_v101.pdf.

\section{NOMENCLATURE}

- Number of nodes: $V=10$.

- Total number of TSs per link: $N=18$.

- Path-loss exponent: $m=4$.

- Euclidean distance between consecutive nodes: $d[\mathrm{~m}]=1$.

- Maximum affordable transmit power per node: $\left(P_{v}\right)_{\max } \cdot[\mathrm{W}]=50$.

- Spatially periodic link scheduling parameter: $T=\{3,4,5,6$, $7,8,9\}$.

Manuscript received November 29, 2013; revised August 1, 2014; accepted September 20, 2014. This work was supported in part by the Republic of Turkey Ministry of National Education, by the Industrial Company Members of the Mobile VCE, by the U.K. Engineering and Physical Sciences Research Council, and by the Research Councils U.K. through the India-U.K. Advanced Technology Center of the European Union under the auspices of the Concerto Project and of the European Research Council's Senior Research Fellow Grant. The review of this paper was coordinated by Prof. S. Chen.

The authors are with the School of Electronics and Computer Science, University of Southampton, Southampton SO17 1BJ, U.K. (e-mail: hy3g09@ ecs.soton.ac.uk; ktkc106@ecs.soton.ac.uk; meh@ecs.soton.ac.uk; lh@ecs. soton.ac.uk).

Color versions of one or more of the figures in this paper are available online at http://ieeexplore.ieee.org.

Digital Object Identifier 10.1109/TVT.2014.2360361
- Initial battery energy per node: $E_{v}[\mathrm{~J}]=5000$.

43

- Spectral noise power density: $N 0[\mathrm{dBm} / \mathrm{Hz}]=1$.

- Power amplifier inefficiency: $\alpha=0.01$ [26].

- Set of all directed links: $\mathcal{L}$.

- A directed link spanning from transmitter $i$ to receiver $j: l_{i, j}$

- Set of all sensor nodes: $\mathcal{V}$.

- Network topology incidence matrix: A.

- Emerging link of node $v: l \in \mathcal{O}(v)$.

- Incoming link of node $v: l \in \mathcal{I}(v)$.

- Network-channel-gain matrix: G.

- Fading gain of the link between transmitter $i$ and receiver $j: 53$ $H_{i, j}=\left|h_{i, j}\right|^{2}$.

- NL: $T_{\text {net }}$.

- Reciprocal of NL: $z$.

- Transmission rate of link $l$ in TS $n: r_{l, n}$.

- Transmit power of link $l$ in TS $n: P_{l, n}$.

- Logarithm of the transmit power of link $l$ in TS $n: Q_{l, n}=59$ $\log \left(P_{l, n}\right)$.

- A set of dual variables for energy conservation constraint in 61 (5): $\Omega$.

- A set of dual variables for transmission rate constraint in (4): $\Psi .63$

- A set of dual variables for transmit power constraint in (6): $\vartheta$. 64

- A set of dual variables for flow constraint in (3): $\boldsymbol{\mu}$.

- Convergence tolerance of the iterative algorithm: $\epsilon=10^{-5}$. 66

\section{INTRODUCTION}

A wireless sensor network (WSN) is composed of a large number 68 of nodes that monitor physical and environmental conditions and pass 69 their accumulated data through the network to a sink node. There are 70 numerous attractive applications for WSNs, including, for example, 71 designing intelligent highways, controlling air pollution, providing 72 remote health assistance for disabled or elderly people, monitoring 73 river level variations, etc. Each of these applications may be composed 74 of many sensor nodes, each of which consumes considerable amount 75 of energy with sensing, communication, and data processing activities. 76 Since each sensor node drains its limited energy supply as time elapses, 77 the network lifetime (NL) is a crucial metric for these applications and 78 has a major impact on the achievable performance of WSNs. Hence, 79 we aim for analyzing and optimizing the NL of the WSNs under 80 different channel conditions.

The NL defines the total amount of time during which the network is 82 capable of maintaining its full functionality and/or achieves particular 83 objectives during its operation, as exemplified in [1] and [2]. Specifi- 84 cally, the authors of [3]-[5] defined the expiration of the NL as the time 85 instant at which a certain number of nodes in the network depleted 86 their batteries. As a further example, the NL was defined in [6] as 87 the lifetime of the specific sensor node associated with the highest 88 energy consumption rate, whereas the authors of [7]-[9] considered 89 the lifetime of the network to be expired at the particular instant, when 90 the first node's battery was depleted. The NL in [8] was also defined 91 as the instant when the first data collection failure occurred. In this 92 paper, the NL is deemed to be expired, when at least one of the nodes 93 fails due to its discharged battery. Therefore, extending the lifetime 94 of a single node becomes an important and challenging task due to 95 the battery-dependent characteristics of the wireless sensor nodes. 96 This common NL definition is used in this paper since we consider 97 
98 a network of linearly connected sensor nodes, where a single node's 99 failure may destroy the entire string topology of nodes and, hence, 100 the information of the source cannot be relayed to the sink. When 101 considering the energy dissipated at a sensor node, the battery life 102 is predominantly related to the node's communication activity, where 103 the transmission rate and power must be optimized, while taking into 104 account the battery capacity, the efficiency of the power amplifiers, the 105 receiver and transmitter circuit energy consumption, and other physical 106 layer parameters, including the modulation and coding schemes, the 107 attainable coding gain, the path loss, and so on.

108 It is widely recognized that transmission at a high transmission rate 109 requires the use of high transmit power, which potentially leads to 110 strong interference among the transmission links [10]. Therefore, the 111 battery depletion of an individual sensor node may become inevitable; 112 hence, the NL may be reduced. However, in large networks, spatial 113 reuse may be adopted for improving the attainable transmission rates at 114 the cost of imposing interference on the network [11]. In this case, link 115 scheduling [12] and multiple-access schemes [13] play a significant 116 role in coordinating the resultant interference. More explicitly, we will 117 demonstrate that scheduling weakly interfering links simultaneously 118 allows the network to maintain a given sum rate at a reduced per-node 119 transmit power, which hence extends the battery life of the nodes and 120 the NL [10]. This is one of the methods routinely employed for taking 121 advantage of spatial reuse to control the level of interference imposed 122 on the network [11]. This method extends the NL since mitigating 123 the interference imposed implies that each transmission requires less 124 power. Therefore, intelligent scheduling should carefully balance the 125 number of simultaneous active links and their transmission duration 126 to keep the required transmit power at a minimum. Furthermore, 127 multihop relaying [14] is capable of conserving the energy of the 128 source node $(\mathrm{SN})$ since intermediate nodes may be employed for 129 reducing the transmission power necessary for maintaining a given 130 end-to-end rate. Hence, we consider the joint optimal design of the 131 transmission rate, transmission power, and scheduling to maximize the 132 NL of energy-constrained WSNs.

133 There is a paucity of contributions in the literature on the issue of 134 cross-layer NL optimization in the context of WSNs. Hoesel et al. 135 [15] proposed a cross-layer approach for jointly optimizing the 136 medium access control (MAC) and routing layer to maximize the 137 NL. Chen and Zhao [8] proposed an efficient MAC protocol that 138 relies both on the channel state information and on the MAC's 139 knowledge of the residual energy to maximize the NL. In [16], 140 Kwon et al. investigated the NL maximization problem of WSNs, 141 which jointly considers the physical layer, the MAC layer, and the 142 routing layer in conjunction with the transmission success probability 143 constraint. Additionally, the tradeoff between NL maximization and 144 application performance was studied in [17] by using cross-layer 145 optimization. A similar study also investigated the tradeoff between 146 the energy consumption and application-layer performance with the 147 aid of cross-layer optimization of WSNs [18]. Another cross-layer ap148 proach conceived for maximizing the NL was proposed in [19], where 149 MAC-aware routing optimization schemes were designed for WSNs 150 that are capable of multichannel access. In [20], Li et al. invoked 151 random linear network coding for the lifetime maximization of wire152 less networks within a fixed-rate system for communicating over both 153 additive white Gaussian noise (AWGN) and Rayleigh fading channels. 154 A different approach to NL maximization was introduced in [21], 155 where both contention and sleep control probabilities of the sensor 156 nodes were utilized for formulating the NL maximization problem, 157 while guaranteeing both the required throughput and the signal-to158 interference-plus-noise ratio (SINR) requirements. Najimi et al. [4] 159 proposed a node selection algorithm for balancing the energy usage of 160 the sensors in a fixed-mode cognitive sensor network. A similar idea

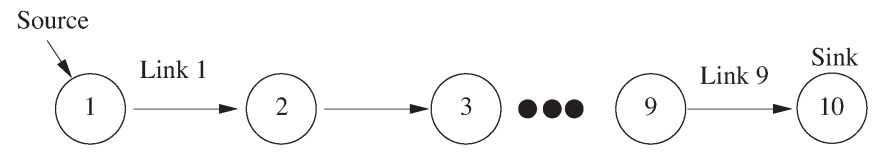

Fig. 1. String topology with $V=10$ nodes, including an SN and a DN.

to that of the optimal control approach invoked for maximizing the NL 161 with the aid of a carefully selected routing probability was exploited 162 in [9], where all the sensors were configured to deplete their energy 163 exactly at the same time for lifetime maximization. Another similar 164 study advocating an effective transmission scheme was proposed in 165 [22], where both the maximum possible energy efficiency and the best 166 possible energy balancing were maintained with the aid of ant colony 167 optimization.

However, all related work aforementioned considers either non- 169 adaptive, i.e., fixed-mode system, or nonfading channel characteristics. 170 An adaptive system conceived for NL maximization was studied by 171 Wang et al. [23], who considered only an interference-free scenario 172 for an AWGN channel by employing the Karush-Kuhn-Tucker (KKT) 173 optimality conditions [24] to the optimal time-division multiple-access 174 (TDMA) NL maximization problem of [12] to derive the analytical 175 expressions of the optimal NL. Madan et al. [12] considered an 176 interference-limited scenario relying on an adaptive system, operating 177 in an AWGN channel, but the impact of the fading channel charac- 178 teristics on the NL was not presented. Wang et al. [23] obtained a 179 closed-form solution for a specific network topology. By contrast, a 180 generalized string network topology consisting of an arbitrary number 181 of nodes is considered in our treatise, where we employ the KKT 182 optimality conditions for obtaining the optimal solution to the NL 183 maximization problem using closed-form expressions. Therefore, we 184 are able to derive analytical expressions of the globally optimal NL for 185 a string network operating in an interference-limited scenario, while 186 communicating either over an AWGN or over fading channels for 187 a given link schedule. Furthermore, the maximum NL, the energy 188 dissipation per node, the average transmission power per link, and the 189 lifetime of all nodes in the network may be obtained. We quantify how 190 the maximum NL decreases as a function of the fading statistics due to 191 the poor channel conditions. Furthermore, it is demonstrated that given 192 a certain network sum rate, the simultaneous scheduling of weakly 193 interfering links benefits from the associated spatial reuse by allowing 194 each node to transmit at a lower rate, which requires a reduced 195 transmission power and hence results in a higher NL. Against this 196 backdrop, the novel contributions of this paper can be summarized as 197 follows.

198

1) The KKT optimality conditions [24] are invoked for deriving 199 the analytical expressions of the globally optimal NL for an 200 interference-limited string topology.

2) In addition to the line-of-sight (LOS) AWGN channel model, 202 the non-LOS Rayleigh block-fading channel model is adopted 203 for studying the effects of fading on the NL.

204

3) The maximum NL is evaluated, and the energy dissipation per 205 node, the average transmission power per link, and the lifetime 206 of all nodes in the network are quantified for a given link 207 schedule and source rate in both LOS AWGN and non-LOS 208 Rayleigh block-fading channels.

4) The substantial effect of the distance among the consecutive 210 nodes on the NL is also analyzed for lower source rates, when 211 operating in a Rayleigh fading channel. The impact of the inter- 212 ferers is also investigated in the context of higher source rates. 213

The remainder of this paper is organized as follows. Section II de- 214 scribes our system model and the constraints of the optimization 215 problem considered. Our problem formulation and solution approach 216 


\begin{tabular}{|c|c|c|c|c|c|c|c|c|c|c|c|c|c|c|c|c|c|c|}
\hline Slots & 1 & 2 & 3 & 4 & 5 & 6 & 7 & 8 & 9 & 10 & 11 & 12 & 13 & 14 & 15 & 16 & 17 & 18 \\
\hline$l_{1-2}$ & • + & & & $\bullet$ & & & - & & & $\stackrel{\bullet}{+}$ & & & - & & & • & & \\
\hline$l_{2-3}$ & & $\cdot$ & & & • & & & $\bullet$ & & & + & & & $\bullet$ & & & $\bullet$ & \\
\hline$l_{3-4}$ & & & • & & & $\bullet$ & & & $\bullet$ & & & $\bullet$ & & & $\bullet$ & & & $\bullet$ \\
\hline$l_{4-5}$ & $\bullet$ & & & • + & & & $\bullet$ & & & • & & & • & & & • & & \\
\hline$l_{5-6}$ & & • & & & + & & & • & & & • & & & $\bullet$ & & & • & \\
\hline$l_{6-7}$ & & & • & & & $\bullet$ & & & • & & & • & & & $\bullet$ & & & • \\
\hline$l_{7-8}$ & $\bullet$ & & & $\bullet$ & & & • + & & & $\bullet$ & & & $\bullet$ & & & • & & \\
\hline$l_{8-9}$ & & • & & & • & & & • & & & • & & & • & & & • & \\
\hline$l_{9-10}$ & & & • & & & $\bullet$ & & & $\bullet$ & & & • & & & • & & & • \\
\hline
\end{tabular}

Fig. 2. Spatially periodic link schedule with time sharing parameter $T=3$ and $T=9$ when $N=18$ and $V=10$.

217 are presented in Section III, and our numerical results are shown in 218 Section IV. Our conclusions are provided in Section V.

\section{SYSTEM MODEL}

220 Here, we first describe the network model, ${ }^{1}$ which relies on a string 221 topology. $^{2}$ Second, we detail our transceiver model in Section II-B, 222 where we evaluate the NL for transmission over both AWGN and 223 block-fading channels. Moreover, our transmission scheduling strategy 224 is also described and exemplified at the end of Section II-B.

\section{A. Network Model}

226 We consider a string topology composed of $V$ sensor nodes, where 227 the SN and the destination node (DN) are linearly connected by 228 intermediate nodes. An example of this string topology for $V=10$ 229 is shown in Fig. 1; hence, the number of links is $L=V-1=9$.

230 Each link is unidirectional, and the antenna of each node is om231 nidirectional. The network can be modeled as a directed graph $\mathcal{G}=$ $232\{\mathcal{V}, \mathcal{L}\}$, where $\mathcal{V}=\{1,2,3, \ldots, V\}$ is the set of all sensor nodes, 233 and $\mathcal{L}=\left\{l_{1,2}, l_{2,3}, \ldots, l_{V-1, V}\right\}$ is the set of all directed links in 234 the network. Here, $l_{i, j}$ represents the directed link spanning from the 235 transmitter node $i$ to receiver node $j$. Therefore, the topology can be 236 modeled with the aid of an incidence matrix of the graph $\mathcal{G}$ given by $237 \mathbf{A} \in \mathbf{R}^{|\mathcal{V}| \times|\mathcal{L}|}$. The entries $a_{v, l}$ of $\mathbf{A}$ are given by

$$
a_{v, l}= \begin{cases}1, & \text { if } v \text { is the transmitter of link } l \\ -1, & \text { if } v \text { is the receiver of link } l \\ 0, & \text { otherwise. }\end{cases}
$$

238 We consider a single commodity flow. Therefore, by the conser239 vation of flow, the constraint $\sum_{l \in \mathcal{O}(v)}\left(\sum_{n=1}^{N} r_{l, n}\right)=\sum_{l \in \mathcal{I}(v)} \times$ $240\left(\sum_{n=1}^{N} r_{l, n}\right)$ may be written for each node $v$ in the absence of an 241 external source or sink, where $N$ is the total number of time slots 242 (TSs) per TDMA frame, and $r_{l, n}$ is the transmission rate of link $l$ in

\footnotetext{
${ }^{1}$ Our network model is a centralized one, where the sink node is assumed to be a control center.

${ }^{2} \mathrm{~A}$ string topology is chosen since, in this simple scenario, the effect of transmission variables on the NL can be explicitly exposed and analyzed. Our string topology scenario is also capable of providing insights concerning a randomly distributed network with many nodes since a specific set of nodes can be assumed to constitute a single route of the randomly distributed network.
}

TS $n$. Additionally, $l \in \mathcal{O}(v)$ denotes the emerging link, and $l \in \mathcal{I}(v) 243$ represents the incoming link of node $v$.

\section{B. Channel Model and MAC Layer Scheme}

In each TS $n$, each node can only act as a transmitter or a receiver. 246 Each transmitter is only allowed to communicate with a single receiver, 247 which cannot receive from other nodes in the same TS. This is due to 248 the half-duplex nature of the transceivers, where nodes communicate 249 on the same shared wireless channel. The channel gain of the link 250 between transmitter $i$ and receiver $j$ is given by $G_{i, j}=1 /\left(d_{i, j}\right)^{m}, 251$ where $d_{i, j}$ is the distance between nodes $i$ and $j$, whereas the path- 252 loss exponent is $m=4$. These channel gains are arranged into a 253 network-channel-gain matrix denoted by $\mathbf{G}$. Each node $v$ is capable 254 of transmitting at a power less than the maximum power of that node 255 denoted by $\left(P_{v}\right)_{\max }$. The total energy dissipation at a node cannot 256 exceed the initial battery energy of that node. No node is allowed to 257 simultaneously transmit multiple data packets, and the link quality is 258 defined by the SINR.

The LOS AWGN channel is modeled by a certain propagation path- 260 loss law and a fixed noise power at the receivers. Given a specific link 261 $l$, the SINR is denoted by $\Gamma_{l}$ in the AWGN channel model. The maxi- 262 mum achievable rate per unit bandwidth is $r_{l}=\log \left(1+K \cdot \Gamma_{l}\right)$ given 263 in nats/s/Hz, where $K=-1.5 / \log (5 \mathrm{BER})$ [25], and BER represents 264 the target bit error ratio (BER) required by the system. Therefore, the 265 SINR is given by [13] $\Gamma_{l_{i, j}, n}=G_{i, j} P_{i, n} /\left(\sum_{i^{\prime} \neq i} G_{i^{\prime}, j} P_{i^{\prime}, n}+N_{0}\right), 266$ where $P_{i, n}$ is the transmission power of node $i$ in TS $n$. Furthermore, 267 $K$ is assumed to incorporate the coding gain and any other gain 268 factors, which is a suitable model for $M$-ary quadrature amplitude 269 modulation (MQAM) associated with $M \geq 4$ [25]. The factor $K$ is 270 assumed absorbed into the gain matrix $\mathbf{G}$.

On the other hand, when considering fading channels, the channel 272 of each link is modeled as a multiplicative Rayleigh fading channel 273 contaminated by the noise added at the receivers. We consider block 274 fading or quasi-static fading, where the fading gain is kept constant 275 throughout the TDMA frame for the link, which represents slowly 276 fading channels, i.e., low Doppler pedestrian speeds. This requires a 277 modification of the SINR used in AWGN channels, which is formu- 278 lated as [25] $\widetilde{\Gamma}_{l_{i, j}, n}=H_{i, j} G_{i, j} P_{i, n} /\left(\sum_{i^{\prime} \neq i} H_{i^{\prime}, j} G_{i^{\prime}, j} P_{i^{\prime}, n}+N_{0}\right), 279$ where $H_{i, j}=\left|h_{i, j}\right|^{2}$ is the fading gain of the link between transmitter 280 $i$ and receiver $j$. 
282 We assume a link scheduling associated with spatially periodic 283 time sharing [12], where we consider a distance $T$ between links 284 that are transmitting in the same TS, and the link is reactivated after 285 every $T$ TSs. Fig. 2 shows the spatially periodic link scheduling for $286 T=3$ and $T=9$. For $T=3$, at the first $T S$, links $l_{1,2}, l_{4,5}, l_{7,8}$ are 287 simultaneously scheduled, and each link is activated six times in total 288 in TSs of $1,4,7,10,13$, and 16. On the other hand, for $T=9$, each TS 289 has only a single active transmission, and each link is activated twice, 290 as shown in Fig. 2.

\section{Problem Formulation AND Solution}

292 Having discussed the assumptions and constraints in Section II, the 293 NL maximization problem [12] can be formulated as in

$$
\begin{aligned}
& \min . \quad z \\
& \text { s.t. } \mathbf{A}\left(\mathbf{r}_{1}+\mathbf{r}_{2}+\cdots+\mathbf{r}_{N}\right)=\mathbf{s} \cdot N \\
& \left(\frac{N_{0}}{G_{i, j}} \cdot e^{r_{l_{i, j}, n}-Q_{l_{i, j}, n}}\right. \\
& \left.+\sum_{l_{i^{\prime}, j^{\prime}} \in \mathcal{L}_{n}, i^{\prime} \neq i} \frac{G_{i^{\prime}, j}}{G_{i, j}} \cdot e^{r_{l_{i, j}, n}+Q_{l_{i^{\prime}, j^{\prime}, n}}-Q_{l_{i, j}, n}}\right) \\
& -1 \leq 0 \quad \forall n, l \in \mathcal{L}_{n} \\
& \sum_{n=1}^{N}\left(\sum_{l \in \mathcal{O}(v) \cap \mathcal{L}_{n}}\left((1+\alpha) \cdot e^{Q_{l_{i, j}, n}}+P_{\mathrm{ct}}\right)\right. \\
& \left.+\sum_{l \in \mathcal{I}(v) \cap \mathcal{L}_{n}} P_{\mathrm{cr}}\right) \leq z \cdot E_{v} \cdot N \quad \forall v \\
& Q_{l_{i, j}, n} \leq \log \left(\left(P_{i}\right)_{\max }\right), l \in \mathcal{L}_{n} \\
& \mathbf{r}_{n} \geq 0 \quad \forall n \\
& r_{l_{i, j}, n}=0 \quad \forall l \notin \mathcal{L}_{n}
\end{aligned}
$$

294 where (4) has been modified, so that it constitutes a strictly convex 295 constraint. See the Nomenclature list on the first page of this paper for 296 the specific parameters utilized in our simulations.

297 The links that are active in TS $n$ are denoted by the set $\mathcal{L}_{n}$, 298 and $\mathbf{s}=\left[s_{1}, 0, \ldots,-s_{1}\right]^{T}$ is the source rate vector, where the first 299 and last elements are nonzero but the remaining elements are set to 300 zero because the first node is the SN and the last node is the DN, 301 and the other nodes act as relay nodes (RN). The variables of the 302 optimization problem are $z, Q_{l, n}$, and $r_{l, n}$, for $l \in \mathcal{L}_{n}, n=1, \ldots, N$. 303 The vector of rate variables associated with TS $n$ is given by $\mathbf{r}_{n}=$ $304\left[r_{l_{1,2}, n}, r_{l_{2,3}, n}, \ldots, r_{l_{V-1, V}, n}\right]^{T}$. Assuming that the transmitter and 305 receiver circuits do not dissipate energy, we can set $P_{\mathrm{ct}}=0$ and $P_{\mathrm{cr}}=0$, 306 where $P_{\mathrm{ct}}$ and $P_{\mathrm{cr}}$ denote the power required by the transmitter 307 and receiver circuits, respectively. Furthermore, we denote the power 308 amplifier inefficiency as $\alpha$ [26]. The lifetime of a node in the network 309 is denoted by $T_{v}$, which corresponds to the time during which the 310 node runs out of battery. The NL is defined as the time during which 311 at least one node completely drains its battery, i.e., we have $T_{\text {net }}=$ $312 \min _{v \neq V, v \in \mathcal{V}} T_{v}$. The objective function $(\mathrm{OF})$ and the constraints of the 313 optimization problem are as follows.

314 1) Objective function-Minimization of reciprocal of the $N L$ : In (2), we minimize $z$ so that the NL is maximized. Here, we used a minimization technique in our problem. We can rewrite (5) as $\sum_{n=1}^{N}\left(\sum_{l \in \mathcal{O}(v) \cap \mathcal{L}_{n}}\left((1+\alpha) \cdot e^{Q_{l_{i, j}, n}}+P_{\mathrm{ct}}\right)+\right.$ $\left.\sum_{l \in \mathcal{I}(v) \cap \mathcal{L}_{n}} P_{\mathrm{cr}}\right) \leq\left(1 / T_{\text {net }}\right) \cdot E_{v} \cdot N$, and we can multiply 318 the left-hand side of the inequality by $T_{\text {net }}$, but the multiplication 319 of the two optimization variables is in general nonconvex. There- 320 fore, we use a change of variable and minimize $z=1 / T_{\text {net }}, 321$ which keeps the right-hand side of the inequality linear and left- 322 hand side convex.

2) Flow conservation constraint: In (3), using matrix A with 324 entries given by 1 ensures that flow conservation is preserved, 325 and physically, this means that the information generated at the 326 $\mathrm{SN}$ has to arrive at the DN.

3) Transmission rate constraint: We have to satisfy the rate con- 328 straint of our interference-limited scenario for each link of the 329 same TS in (4).

4) Energy conservation constraint: Each sensor node can dissipate 331 at most the initial amount of battery energy, which we set to $5000 \mathrm{~J} .332$ Therefore, in (5), the energy conservation constraint is given for 333 each node.

334

5) Transmit power constraint: Equation (6) represents the transmis- 335 sion power at a node, which has to be less than the maximum 336 affordable transmit power of that node.

6) No transmission: Finally, the transmission rate of nodes that are 338 not scheduled for transmission is set to zero in (8).

The optimization problem is solved for the sake of finding the optimal 340 scheme for transmission over each link for a given link schedule, 341 which is defined by the spatially periodic time sharing discussed in 342 Section II-B. However, to obtain the globally optimal solutions, we 343 wish to show that (2)-(8) represent a convex optimization problem, 344 composed of a convex OF, convex inequality constraint functions, and 345 affine equality constraint functions. It is clear that (3) and (8) are affine, 346 (2) and (5)-(7) are convex, and (4) is strictly convex [24]. Therefore, 347 (2)-(8) define a strictly convex optimization problem that has a unique 348 solution. We can convert the problem into its Lagrangian form and 349 rely on the KKT optimality conditions [24] for deriving the analytical 350 expressions of the globally optimal transmission scheme for the string 351 network topology of Fig. 1.

\section{A. Karush-Kuhn-Tucker Optimality Conditions}

Lets us define the sets of the optimization variables and of the 354 Lagrangian multipliers as $\boldsymbol{R}=\left\{r_{l_{1,2}, 1}, \ldots, r_{l_{V-1, V}, N}\right\}, \boldsymbol{Q}=\left\{Q_{l_{1,2}, 1}, 355\right.$ $\left.\ldots, Q_{l_{V-1, V}, N}\right\}, \boldsymbol{\mu}=\left\{\mu_{1}, \ldots, \mu_{V}\right\}, \boldsymbol{\Psi}=\left\{\psi_{l_{1,2}, 1}, \ldots, \psi_{l_{V-1, V}, N}\right\}, 356$ $\boldsymbol{\Omega}=\left\{\omega_{1}, \ldots, \omega_{V-1}\right\}$, and $\boldsymbol{\vartheta}=\left\{\vartheta_{l_{1,2}, 1}, \ldots, \vartheta_{l_{V-1, V}, N}\right\}$. Thus, the 357 partial Lagrangian of (2)-(8) is given by (9), shown at the bottom of 358 the next page, where $\omega_{v}, \psi_{l_{i, j}, n}$, and $\vartheta_{l_{i, j}, n}, \mu_{v}$ are the dual variables 359 associated with the constraints (3)-(6), respectively. Constraints (7) 360 and (8) are taken into account, when deriving the optimal primal 361 variables.

The KKT conditions for (9) are given by (10)-(16), shown at the 363 bottom of the next page, where $\mathcal{I}^{-1}(l)$ denotes the node associated 364 with the incoming link $l$, and $\mathcal{O}^{-1}(l)$ represents the node associated 365 with the outgoing link $l$. Since the primal problem is convex, if 366 $z, \boldsymbol{R}, \boldsymbol{Q}, \boldsymbol{\Omega}, \boldsymbol{\Psi}, \boldsymbol{\vartheta}, \boldsymbol{\mu}$ represent arbitrary points that satisfy the KKT 367 optimality conditions given by the primal feasibility in (3)-(6), the dual 368 feasibility of (16), the complementary slackness in (13)-(15), and the 369 first-order optimality in (10)-(12), then $z, \boldsymbol{R}, \boldsymbol{Q}$ are primal optimal, 370 and $\boldsymbol{\Omega}, \Psi, \vartheta, \boldsymbol{\mu}$ are dual optimal ${ }^{3}$ with zero duality gap ${ }^{4}$ [24].

\footnotetext{
${ }^{3}$ Optimal solution of the primal (original) problem is expressed as primal optimal and the dual problem provides us a lower bound on the optimal value of the original optimization problem. Hence, the dual optimal is a lower bound on the primal optimal.

${ }^{4}$ The duality gap is defined as the difference between the optimal primal and optimal dual solutions.
} 


\section{B. Problem Solution}

373 From (11) and (12), the optimal values of $\boldsymbol{Q}$ and $\boldsymbol{R}$ in iteration $374(t+1)$ are given by (17) and (18), respectively, shown at the bottom of 375 the next page. Note that, due to the interference terms in (11) and (12), 376 each optimal variable in $\boldsymbol{Q}$ and $\boldsymbol{R}$ is dependent on the other variables 377 of $\boldsymbol{Q}$ and $\boldsymbol{R}$, which implies that they are interdependent, hence re378 quiring a centralized solution approach. ${ }^{5}$ Therefore, the Gauss-Seidel

${ }^{5}$ The calculation of both the transmit power and of the rate of a specific node relies on the prior knowledge gleaned from other nodes, possibly from its interferers. Therefore, a control center is required, which handles the variables of the optimization problem and passes the near-instantaneous values of the variables to each of the individual nodes. Compared with a distributed scheme, this centralized solution will impose delay on the system since operations such as channel estimation are required at the initial stage. The near-instantaneous transmission rate and power values computed by the control center constituted by the sink node should be forwarded to each individual node. Therefore, a nonnegligible delay will be imposed on the reception of the sink node. algorithm [27] is utilized for iteratively updating these variables in a 379 circular fashion.

The dual $\mathrm{OF}$ is defined as the minimum value of the Lagrangian 381 (9) over $z, \boldsymbol{R}, \boldsymbol{Q}$ given by $g(\boldsymbol{\Omega}, \boldsymbol{\Psi}, \boldsymbol{\vartheta}, \boldsymbol{\mu})=\inf _{z, \boldsymbol{Q}} \mathcal{L}(z, \boldsymbol{R}, \boldsymbol{Q}, \boldsymbol{\Omega}, \boldsymbol{\Psi}, 382$ $\boldsymbol{\vartheta}, \boldsymbol{\mu})$, which is a linear problem even if the primal problem is 383 nonconvex. The dual function $g(\boldsymbol{\Omega}, \boldsymbol{\Psi}, \boldsymbol{\vartheta}, \boldsymbol{\mu})$ may be maximized to 384 find a lower bound for the optimal value of the primal problem. Then, 385 we can write the dual problem as follows:

$$
\begin{aligned}
\max _{\boldsymbol{\Omega}, \boldsymbol{\Psi}, \boldsymbol{\vartheta}, \boldsymbol{\mu}} & g(\boldsymbol{\Omega}, \boldsymbol{\Psi}, \boldsymbol{\vartheta}, \boldsymbol{\mu}) \\
\text { s.t. } & \boldsymbol{\Omega} \geq 0, \boldsymbol{\Psi} \geq 0, \boldsymbol{\vartheta} \geq 0
\end{aligned}
$$

which is a linear optimization problem. When the primal problem is 387 convex, this lower bound is tight; therefore, the duality gap is zero. 388 Since the dual problem is continuously differentiable, the gradient 389 ascent algorithm [27] is utilized to solve the maximization problem 390 by simply evaluating a series of closed-form expressions. The gradient 391

$$
\begin{aligned}
& \mathcal{L}(z, \boldsymbol{R}, \boldsymbol{Q}, \boldsymbol{\Omega}, \boldsymbol{\Psi}, \boldsymbol{\vartheta}, \boldsymbol{\mu})=z+\sum_{v=1}^{V-1} \omega_{v} \cdot\left[\sum_{n=1}^{N}\left(\sum_{l \in \mathcal{O}(v) \cap \mathcal{L}_{n}}\left((1+\alpha) \cdot e^{Q_{l_{i, j}, n}}\right)\right)-z \cdot E_{v} \cdot N\right] \\
& +\sum_{n=1}^{N} \sum_{l \in \mathcal{L}_{n}} \psi_{l_{i, j}, n} \cdot\left[\left(\frac{N_{0}}{G_{i, j}} e^{r_{l_{i, j}, n}-Q_{l_{i, j}, n}}+\sum_{l_{i^{\prime}, j^{\prime}} \in \mathcal{L}_{n}, i^{\prime} \neq i} \frac{G_{i^{\prime}, j}}{G_{i, j}} e^{r_{l_{i, j}, n}+Q_{l_{i^{\prime}, j^{\prime}, n}}-Q_{l_{i, j}, n}}\right]-1\right] \\
& +\sum_{n=1}^{N} \sum_{l \in \mathcal{L}_{n}} \vartheta_{l_{i, j}, n} \cdot\left[Q_{l_{i, j}, n}-\log \left(\left(P_{i}\right)_{\max }\right)\right]+\sum_{v=1}^{V} \mu_{v} \cdot\left[\sum_{l \in \mathcal{I}(v)}\left(\sum_{n=1}^{N} r_{l_{i, j}, n}\right)-\sum_{l \in \mathcal{O}(v)}\left(\sum_{n=1}^{N} r_{l_{i, j}, n}\right)\right]
\end{aligned}
$$

$$
\begin{aligned}
& \frac{\partial \mathcal{L}}{\partial z}=1-\sum_{v=1}^{V} \omega_{v}\left(E_{v} \cdot N\right)=0 \\
& \frac{\partial \mathcal{L}}{\partial r_{l_{i, j}, n}}=\mu_{\mathcal{I}^{-1}(l)}-\mu_{\mathcal{O}^{-1}(l)}+\psi_{l_{i, j}, n}\left(\frac{N_{0}}{G_{i, j}} e^{r_{l_{i, j}, n}-Q_{l_{i, j}, n}}+\sum_{l_{i^{\prime}, j^{\prime}} \in \mathcal{L}_{n}, i^{\prime} \neq i} \frac{G_{i^{\prime}, j}}{G_{i, j}} e^{r_{l_{i, j}, n}+Q_{l_{i^{\prime}, j^{\prime}, n}}-Q_{l_{i, j}, n}}\right)=0 \quad \forall l, n \\
& \frac{\partial \mathcal{L}}{\partial Q_{l_{i, j}, n}}=\omega_{\mathcal{O}^{-1}(l)}\left((1+\alpha) e^{Q_{l_{i, j}, n}}\right)+\vartheta_{l_{i, j}, n} \\
& -\psi_{l_{i, j}, n}\left(\frac{N_{0}}{G_{i, j}} e^{r_{l_{i, j}, n}-Q_{l_{i, j}, n}}+\sum_{l_{i^{\prime}, j^{\prime}} \in \mathcal{L}_{n}, i^{\prime} \neq i} \frac{G_{i^{\prime}, j}}{G_{i, j}} e^{r_{l_{i, j}, n}+Q_{l_{i^{\prime}, j^{\prime}, n}}-Q_{l_{i, j}, n}}\right)=0 \quad \forall l, n \\
& 0=\sum_{v=1}^{V-1} \omega_{v} \cdot\left[\sum_{n=1}^{N}\left(\sum_{l \in \mathcal{O}(v) \cap \mathcal{L}_{n}}\left((1+\alpha) e^{Q_{l_{i, j}, n}}\right)\right)-z \cdot E_{v} \cdot N\right] \\
& 0=\psi_{l_{i, j}, n} \cdot\left[\left(\frac{N_{0}}{G_{i, j}} e^{r_{l_{i, j}, n}-Q_{l_{i, j}, n}}+\sum_{l_{i^{\prime}, j^{\prime}} \in \mathcal{L}_{n}, i^{\prime} \neq i} \frac{G_{i^{\prime}, j}}{G_{i, j}} e^{r_{l_{i, j}, n}+Q_{l_{i^{\prime}, j^{\prime}, n}}-Q_{l_{i, j}, n}}\right)-1\right] \forall l, n \\
& 0=\vartheta_{l_{i, j}, n} \cdot\left[Q_{l_{i, j}, n}-\log \left(\left(P_{i}\right)_{\max }\right)\right] \quad \forall l, n \\
& \omega_{v} \geq 0, \psi_{l_{i, j}, n} \geq 0, \vartheta_{l_{i, j}, n} \geq 0
\end{aligned}
$$


392 of the Lagrangian function defines the search directions at the current 393 point. Each dual variable is incremented in the direction of the positive 394 gradient in (19)-(22), shown at the bottom of the page, where $t$ is the 395 iteration index, and $[\cdot]^{+}$denotes $\max (0, \cdot)$. Provided that $\Delta_{\Omega}>0$, $396 \Delta_{\Psi}>0, \Delta_{\vartheta}>0$, and $\Delta_{\mu}>0$ are sufficiently small positive step 397 sizes, the dual variables $\boldsymbol{\Omega}^{t}, \boldsymbol{\Psi}^{t}, \boldsymbol{\vartheta}^{t}$, and $\boldsymbol{\mu}^{t}$ converge to the dual 398 optimal variables $\boldsymbol{\Omega}^{*}, \Psi^{*}, \boldsymbol{\vartheta}^{*}$, and $\boldsymbol{\mu}^{*}$, respectively, as $t \rightarrow \infty$. In 399 our case, the optimization problem shown in (2)-(8) is strictly convex; 400 thus, the duality gap is zero, and the solution is unique.

\section{1 \\ IV. EXPERIMENTAL RESULTS}

402 In our experiments, we use the parameters of $d=1 \mathrm{~m}, \alpha=0.01$ 403 [26], $K=1, N_{0}=1 \mathrm{dBm} / \mathrm{Hz}, E_{v}=5000 \mathrm{~J}^{6},\left(P_{i}\right)_{\max }=50 \mathrm{~W}, N=$ $40418, s_{1}=\{0.2,0.3,0.4,0.5,0.6,0.7\}$ nats $/ \mathrm{s} / \mathrm{Hz} \approx\{0.29,0.43,0.58$, $4050.72,0.87,1.01\}$ bits/s/Hz, $T=\{3,4,5,6,7,8,9\}$, convergence toler406 ance of iterative algorithm $\epsilon=10^{-5}$.

407 Fig. 3 shows the NL versus source rate trends for a fixed link 408 schedule and for various spatially periodic time sharing parameters $409 T$, where the channel in each link is a LOS AWGN channel char410 acterized by fixed noise power. As expected, the NL decays as a 411 function of the source rate, as shown in Fig. 3. This is because a 412 higher source rate requires a higher transmission rate and, hence, 413 higher transmission power. Furthermore, in our model, the weakly 414 interfering nodes are scheduled to transmit simultaneously; hence,

${ }^{6}$ For example, this is the energy storage capacity of an AAA alkaline longlife battery.

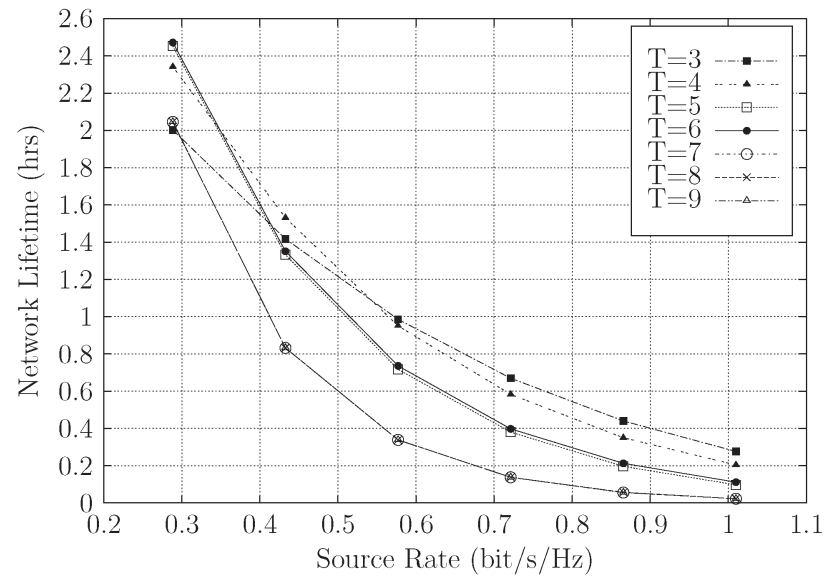

Fig. 3. Network lifetime for different spatially periodic schedules and source rates in the AWGN channel.

each link becomes capable of transmitting at a lower rate, while still 415 satisfying all the transmit requirements of the SN. This necessitates 416 lower transmission power. Using the $T=9$ spatially periodic time 417 schedule of Fig. 2 corresponds to a TDMA scheme since there is only 418 a single transmission in each TS, as shown in Fig. 2. However, since 419 the time frame of Fig. 2 consists of 18 TSs, a specific link is scheduled 420 to transmit twice during the whole time frame. Despite the fact that the 421 $T=9$ link schedule does not impose any interference, it results in the 422 lowest NL according to Fig. 3. Although interference is present in 423

$$
\begin{aligned}
& Q_{l_{i, j}, n}^{t+1}=\log \left[( \mu _ { i } ^ { t } - \mu _ { j } ^ { t } - \vartheta _ { l _ { i , j } , n } ^ { t } ) \cdot \left(\omega_{i}^{t}(1+\alpha)+\sum_{l_{i^{\prime}, j^{\prime}} \in \mathcal{L}_{n}, l_{i^{\prime}, j^{\prime}} \neq l_{i, j}, i^{\prime} \geq i} \psi_{l_{i^{\prime}, j^{\prime}}, n}^{t}\left(\frac{G_{i, j^{\prime}}}{G_{i^{\prime}, j^{\prime}}} \cdot e^{r_{l_{i^{\prime}, j^{\prime}}, n}-Q_{l_{i^{\prime}, j^{\prime}, n}^{t}}}\right)\right.\right. \\
& \left.\left.+\sum_{l_{i^{\prime}, j^{\prime}} \in \mathcal{L}_{n}, l_{i^{\prime}, j^{\prime}} \neq l_{i, j}, i^{\prime}<i} \psi_{l_{i^{\prime}, j^{\prime}}, n}^{t}\left(\frac{G_{i, j^{\prime}}}{G_{i^{\prime}, j^{\prime}}} \cdot e^{r_{l_{i^{\prime}, j^{\prime}, n}^{t}}-Q_{l_{i^{\prime}, j^{\prime}}}^{t+1}}\right)\right)^{-1}\right] \forall l, n \\
& r_{l_{i, j}, n}^{t+1}=\log \left[\frac{\mu_{i}^{t}-\mu_{j}^{t}}{\psi_{l_{i, j}, n}^{t} \cdot\left(\frac{N_{0}}{G_{i, j}}+\sum_{l_{i^{\prime}, j^{\prime}} \in \mathcal{L}_{n}, l_{i^{\prime}, j^{\prime}} \neq l_{i, j}} \frac{G_{i^{\prime}, j}}{G_{i, j}} \cdot e^{Q_{l_{i^{\prime}, j^{\prime}, n}}^{t+1}}\right)}\right]+Q_{l_{i, j}, n}^{t+1} \quad \forall l, n
\end{aligned}
$$

$$
\begin{aligned}
& \omega^{t+1}=\left[\omega^{t}+\Delta_{\omega}\left(\sum_{n=1}^{N}\left(\sum_{l \in \mathcal{O}(v) \cap \mathcal{L}_{n}}\left((1+\alpha) \cdot e^{Q_{l_{i, j}, n}}\right)\right)-z \cdot E_{v} \cdot N\right)\right]^{+} \\
& \psi^{t+1}=\left[\psi^{t}+\Delta_{\psi}\left(\left(\frac{N_{0}}{G_{i, j}} e^{r_{l_{i, j}, n}-Q_{l_{i, j}, n}}+\sum_{l_{i^{\prime}, j^{\prime}} \in \mathcal{L}_{n}, i^{\prime} \neq i} \frac{G_{i^{\prime}, j}}{G_{i, j}} e^{r_{l_{i, j}, n}+Q_{l_{i^{\prime}, j^{\prime}, n}}-Q_{l_{i, j}, n}}\right)-1\right)\right]^{+} \\
& \vartheta^{t+1}=\left[\vartheta^{t}+\Delta_{\vartheta}\left(Q_{l_{i, j}, n}-\log \left(\left(P_{i}\right)_{\max }\right)\right)\right]^{+} \\
& \mu^{t+1}=\left[\mu^{t}+\Delta_{\mu}\left(\sum_{l \in \mathcal{I}(v)}\left(\sum_{n=1}^{N} r_{l_{i, j}, n}\right)-\sum_{l \in \mathcal{O}(v)}\left(\sum_{n=1}^{N} r_{l_{i, j}, n}\right)\right)\right]
\end{aligned}
$$




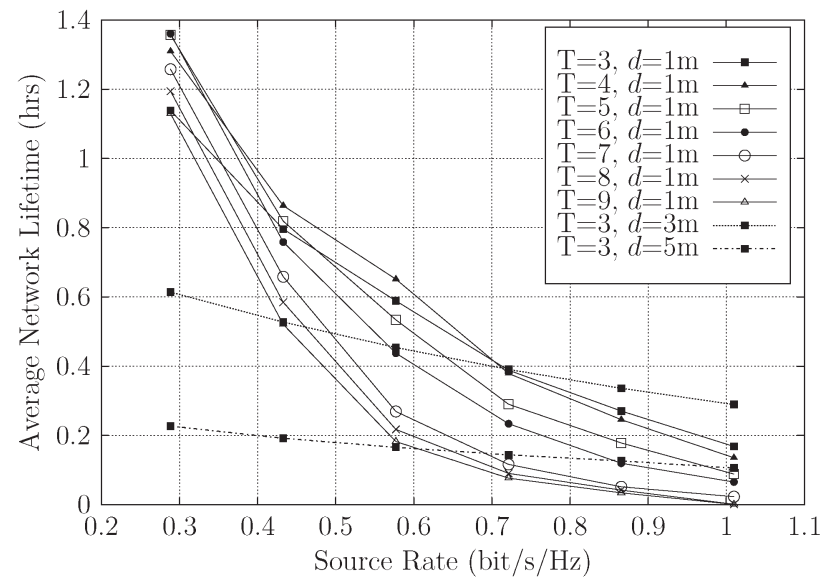

Fig. 4. Network lifetime for different spatially periodic schedules and source rates in a block-fading channel.

424 the $T=3$ scenario since each link can be activated three times more 425 often than in the $T=9$ scenario, each link in the $T=3$ scenario can 426 be activated at lower transmission power, while still satisfying the end427 to-end rate constraint. Therefore, the spatial reuse assisted us in the $428 T=3$ scenario for increasing the NL. We can follow Fig. 2 to find out 429 how many transmissions there are per link for a given value of $T$. For 430 example, the $T=3$ schedule allows a link to be scheduled six times, 431 which requires a reduced transmission rate, since the total source rate 432 that is delivered over different TSs is using six transmissions. From the 433 flow conservation equality constraint of the optimization problem seen 434 in (3), we have $\mathbf{A}\left(\mathbf{r}_{1}+\mathbf{r}_{2}+\cdots+\mathbf{r}_{N}\right)=\mathbf{s} \cdot N$. For example, let us 435 assume that the source rate equals to $0.29 \mathrm{bits} / \mathrm{s} / \mathrm{Hz}$. Then, we obtain $43618 \cdot 0.29=5.22 \mathrm{bits} / \mathrm{s} / \mathrm{Hz}$, which has to be divided into six transmis437 sions, corresponding to a $0.87 \mathrm{bits} / \mathrm{s} / \mathrm{Hz}$ per link transmission rate for $438 T=3$. However, when we have $T=9$, we obtain a $2.61-\mathrm{bits} / \mathrm{s} / \mathrm{Hz}$ 439 per link transmission rate since a link is only activated twice during the 440 whole time frame. Therefore, the transmission rate per link converges 441 to $0.87 \mathrm{bits} / \mathrm{s} / \mathrm{Hz}$ for $T=3$ and $2.61 \mathrm{bits} / \mathrm{s} / \mathrm{Hz}$ for $T=9$. Hence, $442 T=9$ requires three times as much transmission power as $T=3$. 443 The required transmit power in weakly interfering links is quite low 444 compared with that for $T=9$, which is the scenario requiring the 445 highest transmission rate. Hence, again, we surmise that simultaneous 446 scheduling benefits from reduced transmission power due to its re447 duced transmission rate per link. This is because the spatially periodic 448 schedule allows us to schedule more transmissions during the same 449 TS or to activate the same link more than once in different TSs. This 450 explains the steep decay of the NL for $T=9$.

451 When considering the effects of node density on a given fixed link 452 schedule, we expect a network supporting less than $V=10$ nodes to 453 be exposed to less interference. Therefore, the transmission power of 454 each link can be reduced without reducing the end-to-end transmission 455 rate, which results in a higher NL. On the other hand, upon increasing 456 the node density, we expect the NL to decrease since more interferers 457 are introduced, but the same transmission rate is required.

458 Fig. 4 represents the NL versus source rate tradeoff for a fixed 459 link schedule and for various spatially periodic time sharing parameter 460 values of $T$ when each link obeys an independent and identically dis461 tributed Rayleigh block-fading channel. Naturally, the NL was reduced 462 compared with the results of Fig. 3 recorded for an AWGN channel 463 due to requiring higher transmit power to combat the effects of fading. 464 We also analyzed the impact of the internode distance on the NL for 465 the $T=3$-based link schedule, when communicating over a Rayleigh 466 fading channel, as shown in Fig. 4. Increasing the distance between 467 the consecutive nodes substantially reduced the NL, particularly for

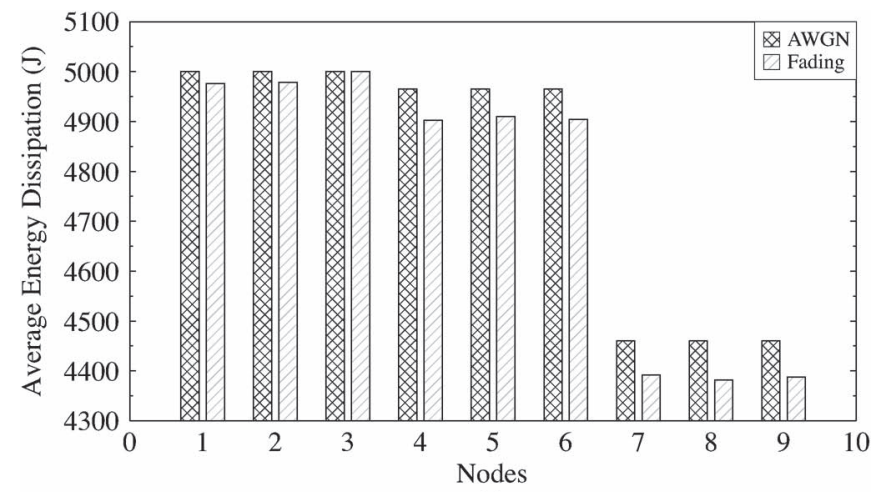

(a)

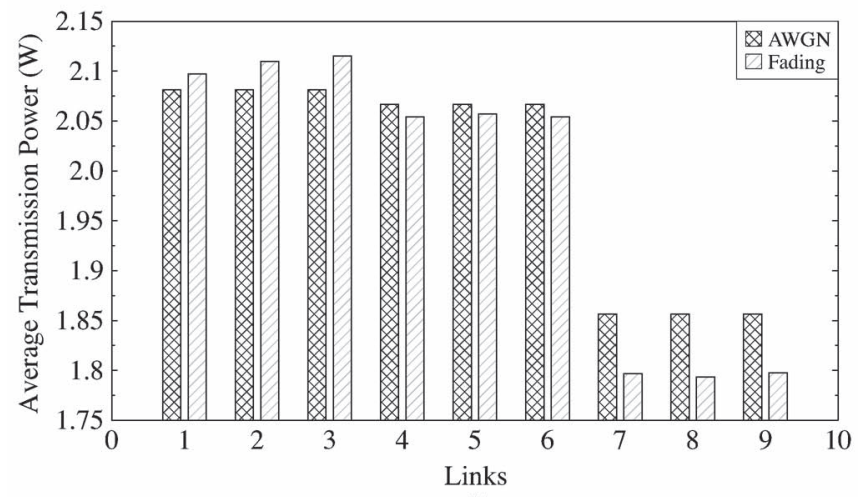

(b)

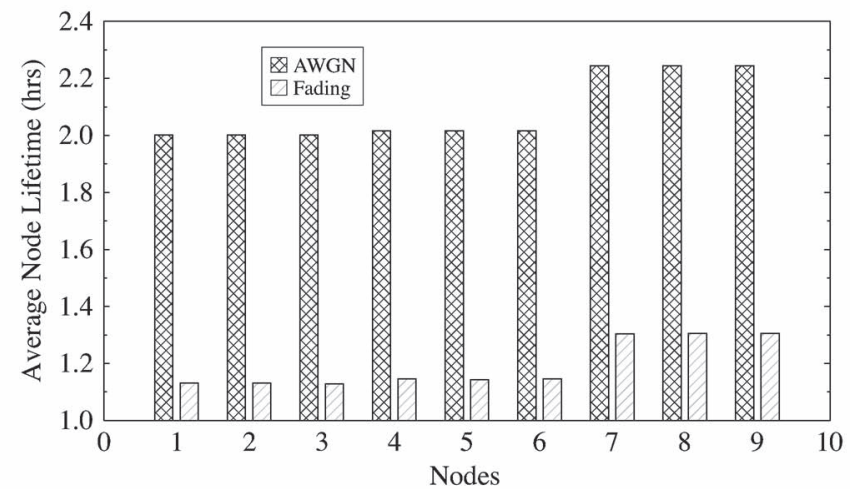

(c)

Fig. 5. Energy dissipation per node, average transmit power per link and lifetime of all nodes in the network in both AWGN and fading channels for the $T=3$ link schedule at a source rate of $0.29 \mathrm{bits} / \mathrm{s} / \mathrm{Hz}$. (a) Energy dissipation per node. (b) Average transmit power per link. (c) Lifetime of all nodes in the network.

lower source rates. However, quite surprisingly, increasing the distance 468 between the consecutive nodes from 1 to $3 \mathrm{~m}$ resulted in an improved 469 NL for higher source rates. This is due to the reduced impact of 470 the interferers located at a higher distance. More explicitly, although 471 the transmit power required had to be increased to satisfy the rate 472 constraint, at the same time the interferers were moved a bit further 473 away. Therefore, the total energy dissipation of the $d=3 \mathrm{~m}$ scenario 474 is still lower than that of the $d=1 \mathrm{~m}$ scenario associated with higher 475 source rates.

476

Furthermore, we comprehensively study the energy dissipation per 477 node, the average transmission power per link, and the lifetime of all 478 sensor nodes in the network. Fig. 5 shows the energy dissipation per 479 node, the average transmission power per link, and the lifetime of all 480 nodes in the network in both AWGN and fading channels for the $T=3481$ link schedule of Fig. 2 at a source rate of 0.29 bits/s/Hz. In the network 482 
483 topology considered, the transmissions from the first three nodes suffer 484 from the highest amount of interference. This is because their receiving 485 nodes are closer to their potential interferers, when compared with 486 any other sets of nodes. Therefore, to satisfy the flow conservation 487 constraints, these nodes must transmit at higher power, as shown in 488 Fig. 5(b). Thus, in an AWGN channel, the first three nodes in the 489 network dissipate their 5000-J initial amount of energy faster than the 490 other nodes since the energy dissipation is proportional to the transmit 491 power, as shown in Fig. 5(a), whereas in the Rayleigh block-fading 492 channel, the third node runs out of battery first, which also determines 493 the lifetime of the WSNs. The required transmit power of the third 494 link is higher than that in the AWGN channel scenario. This increase 495 in transmit power is required to overcome the effect of fading.

496 The average transmit power per link is calculated by summing the 497 transmit power values per link and then by dividing it by the number of 498 TSs that the same link was allowed to transmit. For the first three links 499 operating in the AWGN channel, the required transmission power per 500 link is higher than that of the rest of the links. Since requiring a high 501 transmit power results in dissipating more energy, the lifetime of those 502 nodes is reduced, as shown in Fig. 5(c).

503 Upon comparing the AWGN and fading channel scenarios in Fig. 5, 504 we observe that they follow a similar trend. An observation is that 505 the average transmit power per link of the seventh, eighth, and ninth 506 nodes in Fig. 5(b) is slightly lower for the fading channel than for the 507 AWGN channel. However, interestingly, the average transmit power 508 per link of the third node in Fig. 5(b) recorded for the fading channel 509 is slightly higher than that of the AWGN channel. Therefore, the need 510 for a high transmit power necessitates higher energy dissipation for 511 that particular node. Hence, the NL is reduced, which can also be 512 observed by comparing Figs. 3 and 4. Fig. 3 shows that the NL of 513 the WSN in the AWGN channel recorded for the $T=3$ link schedule 514 and for 0.29 bits/s/Hz source rate is approximately $2 \mathrm{~h}$. By contrast, 515 Fig. 4 shows that the NL of the WSN operating in a Rayleigh fading 516 scenario for the $T=3$ link schedule and for 0.29 -bits $/ \mathrm{s} / \mathrm{Hz}$ source rate 517 indicates approximately an NL of $1.13 \mathrm{~h}$. This earlier node failure of 518 the fading scenario is due to the poor channel conditions, where the 519 fading required higher transmit power in the third node, as shown in 520 Fig. 5(b). Therefore, this earlier node failure shortened the NL of the 521 WSNs in fading channels.

522 To put the given results into context, we apply our analysis to the 523 environmental sensor networks of [28], where the relation between 524 glaciers and climate change was studied. In their work, Martinez et al. 525 [28] transmit data only once per day for a 0.5 -s time slot. In this 526 specific application and considering our results in Fig. 5 for $T=3$ and 527 a source rate of $0.29 \mathrm{bits} / \mathrm{s} / \mathrm{Hz}$, the battery will serve communications 528 for $7200 \mathrm{~s}$, which means that the NL will be around four years and 529 three months in the LOS AWGN channel. We also consider what NL 530 we can achieve if the environmental conditions are more challenging 531 and the channel is exposed to the severe environments mentioned in 532 [28], which may be modeled by a non-LOS Rayleigh block-fading 533 channel. Activating the communication channel once per day in fading 534 conditions will lead us to an NL of around two years and six months.

\section{CONCLUSION}

536 We evaluated the optimal NL in an interference-limited scenario for 537 an optimal transmit rate and power, when considering the so-called 538 spatially periodic time sharing scheme of Fig. 2. The maximization 539 of NL was formulated as a nonlinear optimization problem taking into 540 account the link scheduling, the transmission rates, and transmit power 541 of all active TSs. The original nonlinear problem was converted into 542 a convex optimization problem by employing an approximation of the 543 SINR. We then derived the Lagrangian form of the convex optimiza- tion problem and employed the KKT optimality conditions [24] for 544 deriving analytical expressions of the globally optimal transmit rate 545 and power for our specific network topology. Finally, we obtained the 546 maximum NL for both AWGN and Rayleigh fading channels. Our 547 numerical results illustrated that fading has a detrimental impact on 548 the achievable NL due to the poor channel conditions that require an 549 increased transmit power to combat the effects of the fading. Further- 550 more, the simultaneous scheduling of links that interfere only weakly 551 allowed us to take advantage of spatial reuse, where the activation 552 of simultaneous transmissions at reduced rates necessitates reduced 553 transmission power, which results in extending the NL. From this 554 paper, we can conclude that the choice of scheduling depends on the 555 application since a lower source rate favors infrequent transmissions 556 requiring low transmit power, which do not suffer from interference, 557 when aiming for extending the NL. However, for higher source rates, 558 a higher NL can be achieved by aggressive spatial reuse.

Given the limitations of the centralized solution approach men- 560 tioned in Section III-B, the focus of this paper is on the information 561 delay analysis. We also plan to extend our string topology model to a 562 random network topology, where a single string ( $\mathrm{SN}-\mathrm{DN}$ pair) can be 563 assumed to constitute a single route of the random topology. Nonethe- 564 less, conceiving distributed solutions for avoiding the limitations of our 565 centralized scheme constitutes attractive future research directions. 566

REFERENCES

[1] I. Dietrich and F. Dressler, "On the lifetime of wireless sensor networks," 568 ACM Trans. Sensor Netw., vol. 5, no. 1, pp. 5:1-5:39, Feb. 2009.

[2] W. Liu, K. Lu, J. Wang, G. Xing, and L. Huang, "Performance analysis of 570 wireless sensor networks with mobile sinks," IEEE Trans. Veh. Technol., 571 vol. 61, no. 6, pp. 2777-2788, Jul. 2012.

572

[3] J. Chen, J. Li, and T. Lai, "Trapping mobile targets in wireless sensor 573 networks: An energy-efficient perspective," IEEE Trans. Veh. Technol., 574 vol. 62, no. 7, pp. 3287-3300, Sep. 2013.

[4] M. Najimi, A. Ebrahimzadeh, S. Andargoli, and A. Fallahi, "Lifetime 576 maximization in cognitive sensor networks based on the node selection," 577 IEEE Sensors J., vol. 14, no. 7, pp. 2376-2383, Jul. 2014.

[5] J. Du, K. Wang, H. Liu, and D. Guo, "Maximizing the lifetime of 579 k-discrete barrier coverage using mobile sensors," IEEE Sensors J., 580 vol. 13, no. 12, pp. 4690-4701, Dec. 2013.

[6] H. Salarian, K. Chin, and F. Naghdy, "An energy-efficient mobile-sink 582 path selection strategy for wireless sensor networks," IEEE Trans. Veh. 583 Technol., vol. 63, no. 5, pp. 2407-2419, Jun. 2014.

[7] J. W. Jung and M. Weitnauer, "On using cooperative routing for lifetime 585 optimization of multi-hop wireless sensor networks: Analysis and guide- 586 lines," IEEE Trans. Commun., vol. 61, no. 8, pp. 3413-3423, Aug. 2013. 587

[8] Y. Chen and Q. Zhao, "On the lifetime of wireless sensor networks," IEEE 588 Commun. Lett., vol. 9, no. 11, pp. 976-978, Nov. 2005.

[9] C. Cassandras, T. Wang, and S. Pourazarm, "Optimal routing and en- 590 ergy allocation for lifetime maximization of wireless sensor networks 591 with nonideal batteries," IEEE Trans. Control Netw. Syst., vol. 1, no. 1, 592 pp. 86-98, Mar. 2014.

[10] D. Yuan, V. Angelakis, L. Chen, E. Karipidis, and E. Larsson, "On opti- 594 mal link activation with interference cancelation in wireless networking," 595 IEEE Trans. Veh. Technol., vol. 62, no. 2, pp. 939-945, Feb. 2013.

[11] Z. Yang, Q. Zhang, and Z. Niu, "Throughput improvement by joint relay 597 selection and link scheduling in relay-assisted cellular networks," IEEE 598 Trans. Veh. Technol., vol. 61, no. 6, pp. 2824-2835, Jul. 2012.

599

[12] R. Madan, S. Cui, S. Lall, and A. Goldsmith, "Cross-layer design for 600 lifetime maximization in interference-limited wireless sensor networks," 601 IEEE Trans. Wireless Commun., vol. 5, no. 11, pp. 3142-3152, Nov. 2006. 602

[13] A. Goldsmith, Wireless Communications. Cambridge, U.K.: Cambridge 603 Univ. Press, 2005.

[14] H. Yetgin, K. T. K. Cheung, and L. Hanzo, "Multi-objective routing 605 optimization using evolutionary algorithms," in Proc. IEEE WCNC, Paris, 606 France, Apr. 2012, pp. 3030-3034.

[15] L. Van Hoesel, T. Nieberg, J. Wu, and P. J. M. Havinga, "Prolonging 608 the lifetime of wireless sensor networks by cross-layer interaction," IEEE 609 Wireless Commun. Mag., vol. 11, no. 6, pp. 78-86, Dec. 2004.

610

[16] H. Kwon, T. H. Kim, S. Choi, and B. G. Lee, "A cross-layer strategy 611 for energy-efficient reliable delivery in wireless sensor networks," IEEE 612 Trans. Wireless Commun., vol. 5, no. 12, pp. 3689-3699, Dec. 2006. 
614 [17] H. Nama, M. Chiang, and N. Mandayam, "Utility-lifetime trade-off in self-regulating wireless sensor networks: A cross-layer design approach," in Proc. IEEE ICC, Istanbul, Turkey, Jun. 2006, vol. 8, pp. 3511-3516.

617 [18] J. Zhu, S. Chen, B. Bensaou, and K.-L. Hung, "Tradeoff between life618 time and rate allocation in wireless sensor networks: A cross layer 619 approach," in Proc. 26th IEEE INFOCOM, Anchorage, AK, USA, 620 May 2007, pp. 267-275.

621 [19] S. Ehsan, B. Hamdaoui, and M. Guizani, "Radio and medium access 622 contention aware routing for lifetime maximization in multichannel sensor 623 networks," IEEE Trans. Wireless Commun., vol. 11, no. 9, pp. 3058-3067, 624 Sep. 2012

625 [20] P. Li, S. Guo, and V. Leung, "Maximum-lifetime coding tree for multicast 626 in lossy wireless networks," IEEE Wireless Commun. Lett., vol. 2, no. 3 , pp. 295-298, Jun. 2013.

21] J.-H. Jeon, H.-J. Byun, and J.-T. Lim, "Joint contention and sleep control for lifetime maximization in wireless sensor networks," IEEE Commun. Lett., vol. 17, no. 2, pp. 269-272, Feb. 2013.
[22] X. Liu, "A transmission scheme for wireless sensor networks using ant 631 colony optimization with unconventional characteristics," IEEE Commun. 632 Lett., vol. 18, no. 7, pp. 1214-1217, Jul. 2014.

[23] H. Wang, N. Agoulmine, M. Ma, and Y. Jin, "Network lifetime optimiza- 634 tion in wireless sensor networks," IEEE J. Sel. Areas Commun., vol. 28, 635 no. 7, pp. 1127-1137, Sep. 2010.

[24] S. P. Boyd and L. Vandenberghe, Convex Optimization. Cambridge, 637 U.K.: Cambridge Univ. Press, 2004.

[25] A. Goldsmith and S.-G. Chua, "Variable-rate variable-power MQAM for 639 fading channels," IEEE Trans. Commun., vol. 45, no. 10, pp. 1218-1230, 640 Oct. 1997.

641

[26] M. Albulet, RF Power Amplifiers. Raleigh, NC, USA: SciTech, 2001. 642

[27] D. Palomar and M. Chiang, "A tutorial on decomposition methods for 643 network utility maximization," IEEE J. Sel. Areas Commun., vol. 24, 644 no. 8, pp. 1439-1451, Aug. 2006.

[28] K. Martinez, J. Hart, and R. Ong, "Environmental sensor networks," 646 Computer, vol. 37, no. 8, pp. 50-56, Aug. 2004. 


\section{AUTHOR QUERIES}

AUTHOR PLEASE ANSWER ALL QUERIES

AQ1 = Please provide keywords.

END OF ALL QUERIES 